\title{
Algorithms for Nielsen type periodic numbers of maps with remnant on surfaces with boundary and on bouquets of circles I
}

\author{
by \\ Evelyn L. Hart (Hamilton, NY), Philip R. Heath (St. John's) and \\ Edward C. Keppelmann (Reno, NV)
}

\begin{abstract}
In this paper and its sequel we present a method that, under loose restrictions, is algorithmic for calculating the Nielsen type numbers $N \Phi_{n}(f)$ and $N P_{n}(f)$ of self maps $f$ of hyperbolic surfaces with boundary and also of bouquets of circles. Because self maps of these surfaces have the same homotopy type as maps on wedges of circles, and the Nielsen periodic numbers are homotopy type invariant, we need concentrate only on the latter spaces. Of course the results will then automatically apply to the former spaces as well. The algorithm requires only that $f$ has minimal remnant, by which we mean that there is limited cancellation between the $f_{*}$ images of generators of the fundamental group. These methods often work even when the minimal remnant condition is not satisfied.

Our methodology involves three separate techniques. Firstly, beginning with an endomorphism $h$ on the fundamental group, we adapt an algorithm of Wagner to our setting, allowing us to distinguish non-empty Reidemeister classes for iterates of a special representative map for $h$, which we introduce. Secondly, using techniques reminiscent of symbolic dynamics, we assign key algebraic information to the actual periodic points of this special representative. Finally, we use word length arguments to prove that the remaining information required for the calculation of $N \Phi_{n}(f)$ and $N P_{n}(f)$ can be found with a finite computer search. We include many illustrative examples.

In this first paper we give the tools we need in order to present and give the algorithm for $N P_{n}(f)$. All the tools introduced here will be needed in the sequel where we develop the extra tools needed in order to compute $N \Phi_{n}(f)$.
\end{abstract}

1. Introduction. Let $f: X \rightarrow X$ be a self map of a surface with boundary or a bouquet of circles. In this paper and its sequel we adopt, extend and modify a method of Wagner for finding the Nielsen number of

2000 Mathematics Subject Classification: Primary 55M20.

Key words and phrases: Nielsen number, Nielsen periodic number, fixed point, periodic point, Reidemeister number, surface, punctured disc, surface with boundary, bouquet of circles, wedge of circles, figure eight, remnant.

The first author was supported in part by the Colgate University Research Council. 
maps on these spaces to produce an algorithm for calculating the Nielsen periodic point numbers, $N P_{n}(f)$ and $N \Phi_{n}(f)$, on a large class of self maps on the same spaces Wagner studies. The numbers $N P_{n}(f)$ and $N \Phi_{n}(f)$, which were introduced by Jiang in [13] and studied in [11] and [12], are lower bounds over all maps $g$ homotopic to $f$ for, respectively, the number of periodic points of $g$ of all periods dividing $n$, and the number of periodic points of $g$ of minimal period exactly $n$. The calculation of these numbers is complicated firstly because they are homotopy invariants under homotopies of $f$, rather than homotopies of the iterate $f^{n}$, and secondly because the Nielsen classes of fixed points of various iterates of $f$ interact.

Putting this in its historical context, we remark that the numbers $N P_{n}(f)$ and $N \Phi_{n}(f)$ are, in fact, two different generalizations, from $f$ to iterates of $f$, of the classical Nielsen number $N(f)$ of $f$. This latter number is a lower bound, over all maps $g$ homotopic to $f$, for the number of fixed points of $g$.

Concerning the computation of $N(f)$ for maps on surfaces with negative Euler characteristic (as we study here), McCord remarks in his 1993 survey [20] that maps on these spaces are among those for which this computation is the most difficult, and of course the computation of $N P_{n}(f)$ and $N \Phi_{n}(f)$ on these spaces presents even more of a challenge. In fact, in the years since McCord made his comments about computing $N(f)$ on these spaces its computation has been the focus of much attention. We note firstly that Kelly in [14] produced a useful class of examples of maps on these spaces that factor through the circle. In addition, for the homotopy class of a homeomorphism, he used geometric results of Bestvina and Handel from [1] in [15] to calculate $N(f)$ for these maps of a compact surface with negative Euler characteristic. Next, for a class of maps $f$ that is slightly more general than that considered here, Wagner in [21] introduced techniques from combinatorial group theory into the game, to produce an algorithm for determining $N(f)$. This was continued in [22], in the work of the first author in [5], [6], and by Kim in [17].

For the special case of a space with fundamental group which is free on two generators, results include the following: Firstly, for self maps $f$ on such a space, Wagner's work is extended by Yi [23] and Kim [18] to provide an algorithm for computing $N(f)$. Secondly, for the disc with two holes (the "pants" space), Kelly provides in [14] an algorithm for calculating $\operatorname{Min}(f)=\{|\Phi(g)|: g \simeq f\}$. This, of course, is what $N(f)$ is designed to approximate. Similarly, for maps $f$ of the figure eight, Llibre and Nunes in [19] produce an algorithm for $\operatorname{Min}(f)$. Finally, the fascinating relationship between $\operatorname{Min}(f)$ and $N(f)$ on surfaces is discussed by Kelly in [16].

Wagner's algorithm is in some sense truly innovative, since it turns the philosophy of algebraic topology on its head. The usual technique in this subject is of course to convert a geometric problem into a solvable algebraic 
problem, and then to draw geometric conclusions from the algebraic solution. Wagner of course starts the process in the usual way by transferring the geometric problem to an algebraic one. What she does next is the interesting part. She takes the algebraic information thus obtained and uses it to construct a very special representative self map on a wedge of circles. Since the new map has the same homotopy type as the original one (on the original space), it has the same Nielsen number. Wagner is then able to extract new algebraic information from the geometry of her special representative to compute its Nielsen number. This procedure, then, is algorithmic for a large class of maps on surfaces with boundary and bouquets of circles.

Our aim in writing this paper has been, wherever possible, to extend these tools to the Nielsen type periodic point numbers. Naturally enough the geometry plays a crucial role in the methods we use, and we encounter our first hurdle at exactly this point. The problem is roughly that the geometry of the algebraic iterates and the geometry of the geometric iterates do not coincide. This comes because cancellation can occur when iterating homomorphisms. When this happens the iterate of one of Wagner's special representatives is not the special representative of the iterate. To say it another way, Wagner's algorithm as it stands does not apply to iterates in the way that we need it to. For this reason, it is necessary to make a highly non-trivial extension of her algorithm to cover this situation. Actually, if all that was involved in the computation of $N \Phi_{n}(f)$ and $N P_{n}(f)$ was the computation of the Nielsen classes of iterates, there would be no need either for this paper or its sequel. However, their computation involves a whole host of new algebraic problems that need to be solved, and as part of what we need in order to solve the new problems, it is necessary to preserve the geometry of iterates.

The new problems we will need to solve include the determination firstly of the Reidemeister orbits and then, for the $N P_{n}(f)$ in this first paper, we will need to know which are irreducible. That is, we need to deduce which orbits come up from orbits of lower iterates. For the number $N \Phi_{n}(f)$, we also need to determine the minimum cardinality over all possible sets of $n$ representatives. We will in fact calculate $N \Phi_{n}(f)$ for a number of examples we give in this first paper, but we will not address all of the extra questions it raises until [7]. For completeness, however, we give the definition in Section 2. For both numbers, extending the techniques that work for the computation of $N(f)$ to the new setting is, at times, less than straightforward.

Our methodology involves three separate techniques. Firstly, after replacing the space with a bouquet of circles, we make a highly non-trivial extension of Wagner's algorithm ([21]). This extension allows us to determine completely the Nielsen classes of iterates, their indices, and the corresponding Reidemeister classes from a very special representative, which we 
introduce. The use of this special representative is also the key to our second technique. The point is that while Wagner's algorithm (without modification) can be used to calculate $N\left(f^{k}\right)$ for any $k$, it does not apply to iterates of our special representative and so needs to be extended.

The second part of our methodology, then, uses the special representative to pinpoint the exact location of periodic points of $f$. Using ideas from symbolic dynamics, we are then able to encode location data onto these points that allow us to determine geometrically both the orbits and the geometric reducibility of periodic points. Then in combination with the first technique, this geometric information can be used to find the algebraic orbits of the non-empty Reidemeister classes. (A Reidemeister class is non-empty if it represents an actual periodic point. Otherwise it is called empty.) We will need to consider empty orbits because geometry is not a homotopy invariant. An empty Reidemeister orbit for the special representative of $h$ could correspond to a non-empty orbit for a different representative of $h$.

The third and final part of our methodology pertains exactly to such possibilities. It is necessarily algebraic, and it involves the use of word length arguments to determine which Reidemeister orbits reduce to empty Reidemeister orbits at a lower level. These techniques are completely successful for homomorphisms $h$ with sufficient remnant. For such homomorphisms, therefore, we are able to present an algorithm for determining $N P_{n}(f)$ in this first paper, and $N \Phi_{n}(f)$ in the second for any $f$ that induces $h$. Our methods, by the way, often work even when $h$ does not have the minimum remnant required by the algorithm.

In more detail, but without loss of generality, we let $X$ be a bouquet (i.e. a wedge) of $r$ circles. The fundamental group $\pi_{1}(X)$ is a free group $G=$ $\left\langle a_{1}, \ldots, a_{r}\right\rangle$ on generators $a_{1}, \ldots, a_{r}$. Since these spaces $X$ are EilenbergMacLane complexes of type $K(\pi, 1)$, there is a bijection between the set of homotopy classes of maps $f: X \rightarrow X$ and the set of endomorphisms $h: G$ $\rightarrow G$. Let $h: G \rightarrow G$ be a homomorphism with remnant. From $h$, Wagner in [21] constructs a certain representative, $f$, of the homotopy class corresponding to $h$. This representative has the property that all fixed points are isolated and that, for each $i$, the fixed points on the interior of the $a_{i}$ loop correspond exactly to occurrences of $a_{i}$ or its inverse in the word $h\left(a_{i}\right)$. Her algorithm then allows her, for maps with remnant, to determine not only the precise composition of the Nielsen classes but also the corresponding Reidemeister classes. For a number of reasons, we make a very precise (linearized) version of Wagner's construction, calling it a standard representative for $h$. Of course Wagner's original algorithm can be applied to a standard representative $f$ to determine $N(f)$. However, the relationship between the iterates of $h$ and the fixed points of iterates of $f$ is not quite as straightforward. 
The first step in the study of $N \Phi_{n}(f)$ and $N P_{n}(f)$ is to determine the Nielsen classes of fixed points of the iterates $f^{m}$ of $f$ for all $m \mid n$. Wagner proves in [21] that iterates of maps with remnant have remnant. So we can certainly use her algorithm to determine Nielsen classes of the standard representative $g$ for $h^{n}$. However, what we need is much more complicated. For $n>1$, the standard representative $g$ of $h^{n}$ need not be an iterate of the standard representative $f$ of $h$. Of course $f^{n}$ and $g$ are homotopic, but even when $g$ is of the form $k^{n}$ for some self map $k$, the homotopy need not be induced by a homotopy of $f$ and $k$. In other words, we need to study $f^{n}$ (rather than $g$ ), because $N P_{n}(f)$ and $N \Phi_{n}(f)$ are homotopy invariant under homotopies of $f$ (not of $f^{n}$ ). The problem is that $f^{n}$ often has more fixed points (that is, period $n$ points of $f$ ) than the map $g$, and these extra fixed points are crucial to our second technique for determining orbits of periodic points. We illustrate this in Example 3.1. These additional fixed points occur in pairs. A pair on the $a_{i}$ loop corresponds to occurrences of the letters $a_{i}$ and $a_{i}^{-1}$ that cancel during the calculation of $h^{n}\left(a_{i}\right)$. Therefore we must write the words $h^{n}\left(a_{i}\right)$ in their unreduced form in order to have information about the additional fixed points, which is so crucial to our second technique. So here is the rub. Wagner's proof that her method is algorithmic (and thus finds all Nielsen relations) relies heavily on the homomorphism being in reduced form. Her result as it stands does not guarantee that the method finds all Nielsen equivalences for $f^{n}$. We provide a proof that the very same algorithm does indeed apply to unreduced iterates of homomorphisms.

Our second technique is geometric, and it aims to determine orbits and algebraic reductions of non-empty Reidemeister orbits. In fact there are a number of ad hoc methods that do sometimes (but not always) work (see for example [8] and [7]). We show by example that this is not always the case, and also that our technique works even when ad hoc techniques fail. At its foundation is the very precise construction of the standard representative $f$ of $h$. The idea is simply to build $f$ up from piecewise linear homeomorphisms. The resulting precision of this construction, then, allows us to locate periodic points with any degree of accuracy we may require. We use ideas of symbolic dynamics to assign to each $n$-periodic point of $f$ an " $n$-level address" that encodes information sufficient to determine both the orbit and the minimum period of that point.

The addresses provide an immense amount of information about the geometry of our special representative, which, as we have already pointed out, is not a homotopy invariant. In order to complete our calculations of the $N \Phi_{n}(f)$ and the $N P_{n}(f)$, we need a third technique. It is here that we require the remnant of $h\left(a_{i}\right)$ to have length at least two for each $i$. The point is that while the addresses tell us which non-empty Reidemeis- 
ter orbits reduce to a non-empty Reidemeister orbit of a lower iterate, they do not help us to determine which of the non-empty Reidemeister orbits are reducible to empty Reidemeister orbits at lower iterates. In this first paper where our emphasis is on $N P_{n}(f)$, all we need to know is which such orbits are irreducible. We will have more work to do in [7], in order to compute $N \Phi_{n}(f)$. Our third technique, then, is necessarily purely algebraic. The reduction of a Reidemeister orbit corresponds to a solution to an equation in the fundamental group. When $h$ has remnant of length at least two (Definition 2.10), word length arguments enable us to provide an upper bound on the length of such solutions. The third technique then is to calculate this bound and then defer to a finite computer search to test for solutions of length less than (or equal to) this bound. The finite search then either gives us the reduction or shows us that no such reduction exists. In order to prove that this process is algorithmic for maps with sufficient remnant, we prove that, under such circumstances, we need make only a finite number of such finite searches in order to calculate both $N P_{n}(f)$ and $N \Phi_{n}(f)$.

Throughout, we use examples to illustrate the numerous complications and steps of our algorithm. In all but one case, we need only the free group on two generators to do this. Yet our algorithm applies to any finitely generated free fundamental group. Most of the examples were found by carefully programmed searches using the Magma computer algebra system ([2]).

This first paper is organized as follows. Following this introduction, we give in Section 2 a brief overview of the necessary background for our work, illustrated by an example. In Section 3 we first use an example to motivate the need to extend the proof of Wagner's algorithm to the unreduced form of iterates of $h$. Then we state the theorem that says that Wagner's algorithm does apply to our setting. Because of its technical nature, the proof of this extension is deferred to the appendix in Section 7. In Section 4 we present ideas reminiscent of symbolic dynamics, introducing itineraries and addresses. These concepts are used to determine orbits and to keep track of geometric reductions. The connection between these things and the corresponding Reidemeister classes becomes explicit and algorithmic at this point. Section 5 gives the algebraic word length arguments that allow us to determine when a Nielsen orbit of length less than $n$ at level $n$ reduces algebraically. In Section 6 we present our algorithm for the calculation of $N P_{n}(f)$.

The authors would like to thank the referee for useful comments and detailed observations that have helped us to improve this paper. 
2. Preliminaries. We briefly present the necessary notation and background for Nielsen periodic point theory and for Wagner's algorithm. Details can be found in any of several easily available articles, which we cite as we go.

2.1. Nielsen theory and Nielsen periodic point theory. Any surface with boundary has the same homotopy type as a bouquet of circles. For this reason, we assume for the entire paper that $X$ is a bouquet of circles. Let $f: X \rightarrow X$ be a map. For each positive integer $n$, the $n$th iterate of $f$ is $f^{n}$, and the fixed point set of $f^{n}$ is $\Phi\left(f^{n}\right)=\left\{x \in X: f^{n}(x)=x\right\}$. For $x \in \Phi\left(f^{n}\right)$ we define the period of $x$ to be the smallest positive integer $m \mid n$ such that $x \in \Phi\left(f^{m}\right)$.

The goal of Nielsen fixed point theory is to estimate $M(f)=\min \{\# \Phi(g)$ : $g \sim f\}$. The Nielsen number $N(f)$ of $f$ is a homotopy invariant that is a lower bound for $M(f)$. (See for example [3] and [13].) Nielsen periodic point theory, on the other hand, was developed in order to estimate two other minimum numbers. Let $\operatorname{Per}_{n}(f)$ be the set of all fixed points of $f^{n}$ that have least period $n$, and $M P_{n}(f)=\min \left\{\# \operatorname{Per}_{n}(g): g \sim f\right\}$. Also let $M \Phi_{n}(f)=\min \left\{\# \Phi\left(g^{n}\right): g \sim f\right\}$. The Nielsen periodic numbers $N P_{n}(f)$ and $N \Phi_{n}(f)$ (defined below in Definitions 2.3 and 2.3) are the corresponding homotopy invariant lower bounds. That is, $N P_{n}(f) \leq M P_{n}(f)$ and $N \Phi_{n}(f) \leq M \Phi_{n}(f)$. It is crucial to note that $N P_{n}(f)$ and $N \Phi_{n}(f)$ are homotopy invariants of $f$ and not of $f^{n}$.

We assume familiarity with the concepts of length, depth, and reducibility of both the Nielsen classes, and of the Reidemeister classes for $f^{n}$, as well as for their orbits. These concepts were introduced in Jiang's book [13] and then developed in [11] and [12]. The necessary background information is also explained in detail in the preliminary section of [8] and elsewhere. The set of Nielsen classes of $f^{n}$ is denoted by $\Phi\left(f^{n}\right) / \sim$. For $\mathbf{C}^{n} \in \Phi\left(f^{n}\right) / \sim$, the Nielsen orbit of $\mathbf{C}^{n}$ is the set $\left\{\mathbf{C}^{n}, f\left(\mathbf{C}^{n}\right), \ldots, f^{n-1}\left(\mathbf{C}^{n}\right)\right\} \subseteq \Phi\left(f^{n}\right) / \sim$. Note that these classes need not be distinct. The geometric change of level function, induced by inclusion, is $\gamma_{m, n}: \Phi\left(f^{m}\right) / \sim \rightarrow \Phi\left(f^{n}\right) / \sim$.

Let $\alpha \in \pi_{1}\left(X, x_{0}\right)$. The Reidemeister class for $f^{n}$ containing $\alpha$ is $[\alpha]^{n}$, and the set of all Reidemeister classes for $f_{*}^{n}$ is denoted by $\mathcal{R}\left(f_{*}^{n}\right)$. The usual correspondence between the geometry and the algebra is denoted by $\varrho_{n}=\varrho: \Phi\left(f^{n}\right) / \sim \rightarrow \mathcal{R}\left(f_{*}^{n}\right)$. A Reidemeister class $[\alpha]^{n}$ is called non-empty if it is in the image of $\varrho_{n}$. We use $\left\langle[\alpha]^{n}\right\rangle$ to denote the Reidemeister orbit of $[\alpha]^{n}$.

REMARK 2.1 (Warning about notation). For the Reidemeister action we use the notation of the joint work of the last two authors (in [10]), which is different from, but equivalent to, the notation of [5], [21], and [8]. The Reidemeister equivalence for $f_{*}^{n}$ is given by $\alpha \sim \beta$ if and only if there exists 
a $\delta \in \pi_{1}\left(X, x_{0}\right)$, with $\alpha=\delta \beta f_{*}^{n}\left(\delta^{-1}\right)$. Thus the algebraic change of level function $\iota_{m, n}: \pi_{1}(X) \rightarrow \pi_{1}(X)$ is defined by

$$
\iota_{m, n}(\alpha)=\alpha f_{*}^{m}(\alpha) f_{*}^{2 m}(\alpha) \cdots f_{*}^{n-m}(\alpha) .
$$

It is easy to check that $\iota_{m, n}$ is well defined on Reidemeister classes and so can be considered as a function $\iota_{m, n}: \mathcal{R}\left(f_{*}^{m}\right) \rightarrow \mathcal{R}\left(f_{*}^{n}\right)$. A class $[\alpha]^{n}$ is irreducible if and only if it does not lie in the image of $\iota_{m, n}$ for any proper divisor $m$ of $n$. We note that $\iota_{m, n}$, and thus reducibility, are well defined on orbits, as is essentiality.

We will use the following fact from [8] consistently in the paper without reference to it.

FACT 2.2 (On the nose boosting). The class $[\alpha]^{m}$ at level $m$ boosts to a class $[\beta]^{n}$ at level $n$ if and only if for any representative $\beta$ of $[\beta]^{n}$ there exists a representative $\alpha$ of $[\alpha]^{m}$ for which $\iota_{m, n}(\alpha)=\beta$ in the fundamental group.

Definition 2.3. For each positive integer $n$ we define $N P_{n}(f)=$ $n \#\left(I E O_{n}\right)$, where \# $\left(I E O_{n}\right)$ is the number of irreducible essential Reidemeister orbits of $f$ at level $n$.

Note that the definition takes into account the fact that if there is one periodic point of least period $n$ then there are at least $n$ of them. Before defining $N \Phi_{n}(f)$, we need additional notation. A set of $n$-representatives for $f$ is a collection of Reidemeister orbits at various levels with the property that any essential orbit $\left\langle[\alpha]^{m}\right\rangle$ with $m \mid n$ reduces to some element in this set. The height of such a set is the sum of the depths of all its members.

Definition 2.4. The number $N \Phi_{n}(f)$ is defined to be the minimum height over all sets of $n$-representatives for $f$.

2.2. The standard representative of $h$. Let $G=\pi_{1}(X)=\left\langle a_{1}, \ldots, a_{r}\right\rangle$ be the free group on the generators $a_{i}$, with each $a_{i}$ corresponding to an oriented loop of $X$. Let

$$
\mathcal{G}=\left\{a_{1}, \ldots, a_{r}\right\}
$$

be the set of generators. Given a homomorphism $h: G \rightarrow G$ we define below a special representative map $f: X \rightarrow X$ with $f_{*}=h$, which will allow us to use ideas from symbolic dynamics to study iterates of $f$. Our representative $f$ preserves the base point (the wedge point $x_{0}$ ), and each fixed point is isolated with a location that is easily determined from the words $h\left(a_{i}\right)$ with $a_{i} \in \mathcal{G}$.

We begin as Wagner does. Let $N$ be a small closed neighborhood of $x_{0}$, and require that $f(N)=x_{0}$. Let $a_{i} \in \mathcal{G}$, and use $a_{i}$ to refer to the corresponding oriented loop of $X$ as well. Define $f$ on $a_{i} \backslash N$ by first chopping $a_{i} \backslash N$ into $m_{i}$ pieces of equal length, where $m_{i}$ is the length of the word $h\left(a_{i}\right)$. 
Orient each piece in the same direction as the $a_{i}$-loop. Next, under $f$ send each of the end points of each section to $x_{0}$. Finally, let $Y$ be the open $j$ th section of the $a_{i}$-loop. Define the restriction of $f$ to $Y$ to be one of the two possible linear homeomorphisms from $Y$ onto the loop represented by the $j$ th letter of $h\left(a_{i}\right)$. If the $j$ th letter of $h\left(a_{i}\right)$ is $c \in \mathcal{G}$, then send the segment $Y$ linearly onto $c \backslash x_{0}$ preserving orientation. On the other hand, if the $j$ th letter of $h\left(a_{i}\right)$ is $c^{-1}$ for some $c \in \mathcal{G}$, then send $Y$ linearly onto $c \backslash x_{0}$ reversing orientation.

Notation 2.5. Starting with $a_{1}$ above, we will name the first $m_{1}$ segments, in consecutive order, by the numbers $1, \ldots, m_{1}$. Then for $a_{2}$ we name the next $m_{2}$ segments $m_{1}+1, m_{1}+2, \ldots, m_{1}+m_{2}$ etc.

Definition 2.6. Let $h: G \rightarrow G$ be a homomorphism. The map $f$ defined above is a standard representative $\left({ }^{1}\right)$ for $h$. It depends only on $N, \mathcal{G}$, and the orientations of our loops.

REMARK 2.7. As we will see in Example 3.1, when $f$ is a standard representative for $h$ the iterate $f^{n}$ of $f$ need not be a standard representative for $h^{n}$.

From now on throughout the paper, we will use upper case letters to denote inverses of elements of $G$. Thus if $c$ is a generator of $G, C$ will denote the inverse of $c$.

LEMma 2.8 (Wagner [21]). Let $f$ be a standard representative for $h=$ $f_{*}: G \rightarrow G$, and let $c \in \mathcal{G}$. Then there is a bijection between $\Phi\left(\left.f\right|_{c}\right) \backslash\left\{x_{0}\right\}$ and the occurrences of $c$ and $C$ in the word $f_{*}(c)$. The index of those fixed points associated with the occurrences of $c$ is -1 , and of those with $C$ is +1 . (The index of the base point $x_{0}$ is +1 .)

Making the appropriate generalization of Lemma 2.8 to iterates will allow us later to identify a fixed (periodic) point of $f^{n}$ and its index with the address that describes its location.

ExAmPle 2.9. Let $X$ be the wedge of two circles with $G=\langle a, b\rangle$, and let the homomorphism $f_{*}$ be defined by $f_{*}(a)=a a b$ and $f_{*}(b)=B a$. The graph found in Section 4 illustrates what happens on the $b$ loop. We have $\Phi(f)=$ $\left\{x_{0}, x_{1}, x_{2}, x_{3}\right\}$, where $x_{1}$ and $x_{2}$ are the unique fixed points determined by the first and second $a$ 's in the $a$ loop, and where $x_{3}$ is the unique fixed point determined by the $B$ in the $b$ loop.

$\left({ }^{1}\right)$ There is a small difference between our definition and Wagner's. Because of precise location considerations of the iterates, we require the map to be linear on the pieces; Wagner did not. However, a representative in our sense is also a representative in Wagner's sense. We will not distinguish between them from now on. 
2.3. $W_{x}, \bar{W}_{x}$, maps with remnant, and Wagner's algorithm. In [21], Wagner presents a method (Theorem 2.12 below) for finding Reidemeister equivalences for maps on surfaces with boundary (see also [5]). Wagner proves that for maps with a certain property (having remnant) her method is algorithmic in that it finds all Reidemeister equivalences that are needed to determine the Nielsen number.

The major advantage of the standard representative $f$ for a homomorphism is that for each $x \in \Phi(f)$ there are two easily determined elements of $G$ ( $W_{x}$ and $\bar{W}_{x}$, called Wagner tails), both of which represent the Reidemeister class $\left[W_{x}\right]^{1}=\left[\bar{W}_{x}\right]^{1}$, representing $\left({ }^{2}\right) x$.

A consequence of the notational choice mentioned in Remark 2.1 is that the $W$ 's and $\bar{W}$ 's here are the inverses of those in [21] and [5]. Note in particular, as explained below, that $f_{*}(a)=W_{x}^{-1} a \bar{W}_{x}$.

Let $f(a)=c_{1} \cdots c_{k}$ with each $c_{i} \in \mathcal{G} \cup \mathcal{G}^{-1}$, and let $x$ be a fixed point of $f$ corresponding to some $c_{i}$ with $c_{i} \in\{a, A\}$.

CASE 1: If $c_{i}=a$, then $W_{x}^{-1}=c_{1} \cdots c_{i-1}$ and $\bar{W}_{x}=c_{i+1} \cdots c_{k}$.

CASE 2: If $c_{i}=A$, then $W_{x}^{-1}=c_{1} \cdots c_{i-1} A$ and $\bar{W}_{x}=A c_{i+1} \cdots c_{k}$.

Note that in both cases, $f(a)=W_{x}^{-1} a \bar{W}_{x}$ although this product is in an unreduced form in Case 2. Note that $W_{x}$ determines $\bar{W}_{x}$ because $\bar{W}_{x}=$ $A W_{x} f(a)$, and from this it is clear that $\left[W_{x}\right]^{1}=\left[\bar{W}_{x}\right]^{1}$. Since the base path is chosen to be the constant path at $x_{0}$, the Nielsen class containing $x_{0}$ corresponds to the Reidemeister class [1] ${ }^{1}$. Define $W_{x_{0}}=1=\bar{W}_{x_{0}}$. This definition of the Wagner tails is equivalent to the definition introduced by Fadell and Husseini in [4] using the Fox calculus (also described in [5]).

Wagner calls two fixed points $x$ and $y$ in $\Phi(f)$ directly related if $\left\{W_{x}, \bar{W}_{x}\right\}$ $\cap\left\{W_{y}, \bar{W}_{y}\right\} \neq \emptyset$. Clearly if $x$ and $y$ are directly related they are Nielsen equivalent because $\left[W_{x}\right]^{1}=\left[W_{y}\right]^{1}$. Thus given a sequence of fixed points $x_{1}, \ldots, x_{s}$ for which $x_{i}$ is directly related to $x_{i+1}$ for $i=1, \ldots, s-1$, we can conclude that $x_{1}$ is Nielsen equivalent to $x_{s}$ and $\left[W_{x_{1}}\right]^{1}=\left[W_{x_{s}}\right]^{1}$. So then the notion of being directly related generates an equivalence relation on the set of non-empty Reidemeister classes (those representing actual fixed points of $f$ ). The obvious question is if this equivalence relation is the same as Reidemeister equivalence. That is, are any two Nielsen equivalent fixed points connected by a chain of directly related fixed points?

EXAmple 2.9 (part two). Recall that $f_{*}(a)=a a b$ and $f_{*}(b)=B a$. Wagner's definitions give us the table:

$\left(^{2}\right)$ A fixed point $x$ of $f^{k}$ has a different Reidemeister representative for each iterate of $f^{k}$. To distinguish them, we use superscripts (i.e. 1 here), to indicate the iterate under consideration. 


\begin{tabular}{crrrr}
\hline Fixed point for $f$ & loop & index & $W_{x}$ & $\bar{W}_{x}$ \\
\hline$x_{0}$ & -- & +1 & 1 & 1 \\
$x_{1}$ & $a$ loop & -1 & 1 & $a b$ \\
$x_{2}$ & $a$ loop & -1 & $A$ & $b$ \\
$x_{3}$ & $b$ loop & +1 & $b$ & $B a$ \\
\hline
\end{tabular}

We conclude that $x_{0}$ and $x_{1}$ are both represented by $[1]^{1}$ and that $x_{2}$ and $x_{3}$ are both represented by $[A]^{1}$. The reader will see that the table then determines a partition of $\Phi(f)$ into $\left\{x_{0}, x_{1}\right\}$ and $\left\{x_{2}, x_{3}\right\}$. From the theory so far, we cannot tell if all four points are in the same Nielsen class. That is, we do not know yet if $[A]^{1}=[1]^{1}$.

The fact is that for some maps, the table of Wagner tails does not allow us to determine all Nielsen equivalences (see Example 3.10 of [21]). The beauty of Wagner's algorithm is that it gives an easily checked algebraic condition on the homomorphism for which the Wagner tails completely determine the reduced form of the Reidemeister trace (and hence of course the Nielsen number). The class of maps in question are those with remnant; that is, roughly speaking, those maps that have limited cancellation in products of the $f_{*}\left(a_{i}\right)$ and their inverses.

In order to define remnant, we define a standard decomposition of each of the words $f_{*}(a)$ for $a \in \mathcal{G}$. So let $a \in \mathcal{G}$, and define $U_{a}, R_{a}, V_{a} \in G$ to be the unique words with the following three properties. Firstly, $f_{*}(a)=U_{a} R_{a} V_{a}$, secondly, except for the possibility that $U_{a}, R_{a}$, and $V_{a}$ may be equal to 1 , the product $U_{a} R_{a} V_{a}$ is reduced in $G$. Thirdly, $R_{a}$ is the longest reduced subword of $U_{a} R_{a} V_{a}$ for which no subword of $R_{a}$ cancels in any of the products $f_{*}(a) f_{*}(b)$ and $f_{*}(b) f_{*}(a)$ for all $b \in \mathcal{G}$, and $f_{*}(a) f_{*}\left(b^{-1}\right)$ and $f_{*}\left(b^{-1}\right) f_{*}(a)$ for all $b \in \mathcal{G} \backslash\{a\}$.

Definition 2.10. Let $f: X \rightarrow X$ be a standard representative of a homomorphism $h: G \rightarrow G$ with $G=\pi_{1}\left(X, x_{0}\right)$ a finitely generated free group. Let $a \in \mathcal{G}$. If $R_{a} \neq 1$ in $G$, then the word $f_{*}(a)$ is said to have remnant, and $R_{a}$ is said to be the remnant of $f_{*}(a)$. If for each $a \in \mathcal{G}$ we have $R_{a} \neq 1$, then we say that $f$ has remnant. The map $f$ has minimum remnant $k$ if $k=\min \left\{\left|R_{a}\right|: a \in \mathcal{G}\right\}$, where $\left|R_{a}\right|$ is the length of $R_{a}$.

We can use the following result of Wagner "as is, where is."

Lemma 2.11 (Wagner [21]). Suppose that $f$ is a map on a bouquet of circles that has remnant. Then all iterates of $f$ have remnant as well.

The next theorem is the main result of [21]. A proof with simplified notation can be found in [5]. The proof consists of showing that the Wagner tails find all Nielsen equivalences for a standard representative of a homomorphism that has remnant. 
THEOREM 2.12 (Wagner [21]). Let $f$ be a standard representative for a homomorphism $h: G \rightarrow G$. Suppose also that $f$ has remnant. For $x, y \in$ $\Phi(f), x$ is Nielsen equivalent to $y$ iff there exists a sequence of fixed points $\left\{x_{i}\right\}_{i=1}^{n}$ for some $n$, with $x_{1}=x, x_{n}=y$, and $x_{i}$ directly related to $x_{i+1}$ for $i=1, \ldots, n-1$.

EXAMPLE 2.9 (part three). The map $f$ given by $f_{*}(a)=a a b$ and $f_{*}(b)=$ $B a$ has remnant since $R_{a}=a a$ and $R_{b}=a$. By Theorem 2.12, the table of Wagner tails determines that $[1]^{1} \neq[A]^{1}$. Thus there are two Nielsen classes. Note, however, that both classes are inessential so that $N(f)=N P_{1}(f)=$ $N \Phi_{1}(f)=L(f)=0$.

3. Iterates and the new questions for $N P_{n}(f)$. We are finally ready to start our discussion of the Nielsen type numbers $N P_{n}(f)$ and $N \Phi_{n}(f)$ on bouquets of circles. We first present an example that illustrates the fact, stated in Remark 2.7, that the $n$th iterate of a standard representative for $h$ is not necessarily a standard representative for $h^{n}$. We then indicate the difficulties that this causes for us and the reason that we must extend Theorem 2.12 to unreduced settings. In addition we discuss the role of the Wagner tails in determining the boosting of Reidemeister classes, and we demonstrate the limitations of the Wagner tails in determining Reidemeister orbits. The determination of both boostings and Reidemeister orbits is crucial for the calculation of $N P_{n}(f)$ and $N \Phi_{n}(f)$.

EXAmPLE 3.1. Let $G=\langle a, b\rangle$, and let $h(a)=a b a$ and $h(b)=b A$. Then $h$ has remnant, and by Lemma 2.11 of Wagner, so does $h^{2}$. Let $f$ be a standard representative for $h$. We consider the fixed points of $f^{2}$. The construction of $f$ involved cutting $a \backslash N$ into three equal pieces. We label the three intervals 1,2 , and 3 with order increasing in the direction of the orientation of $a$. Similarly we use 4 and 5 to label the two pieces of $b \backslash N$. Note that $f$ sends 2 once around the $b$ loop, preserving orientation. Thus $f^{2}$ sends 2 forward around the $b$ loop, and then backward (reversing orientation) around the $a$ loop. Because 2 is in the $a$ loop, the piece 2 contains a fixed point of $f^{2}$. Similarly, $f$ sends 3 forward around $a$, so $f^{2}$ sends 3 first forward around $a$, then forward around $b$, and finally forward around $a$ again. We will make this construction more precise when we define addresses in Section 4. So then, 3 contains two fixed points of $f^{2}$. By applying the same reasoning to 1,4 , and 5 , we see that $f^{2}$ has eight fixed points, including the base point. We name the fixed points $x_{0}, \ldots, x_{7}$, with $x_{1}, \ldots, x_{5}$ in the $a$ loop with subscripts increasing in the order of the orientation of $a$. The fixed points $x_{6}$ and $x_{7}$, then, are on the $b$ loop with subscripts increasing in the order of the orientation of $b$.

On the other hand, the homomorphism $h^{2}$ is given by $h^{2}(a)=a b a b b a$ (canceling $A a$ in $f^{2}(a)=a \overline{b a b A a b a)}$ and $h^{2}(b)=b A A B A$. So a standard 
representative for $h^{2}$ will have only six fixed points. Now of course $f^{2}$ is homotopic to the standard representative for $h^{2}$. But $N P_{2}(f)$ and $N \Phi_{2}(f)$ are invariant under homotopies of $f$, but they are not necessarily invariant under homotopies of $f^{2}$. Confusing this point is a common mistake, but applying a homotopy to the iterate of $f$, rather than to $f$ itself, can change the Nielsen periodic point numbers $\left({ }^{3}\right)$. So while it is tempting to ignore the fixed points $x_{3}$ and $x_{4}$ of $f^{2}$ (because they are Nielsen equivalent and have indices +1 and -1 ), we cannot do this, since it would change the two Nielsen type numbers $N P_{n}(f)$ and $N \Phi_{n}(f)$. In addition, as we will see later, we need these points because they are in geometric orbits of other fixed points of $f^{2}$.

The point is that in order to use our methods to compute the numbers $N P_{n}(f)$ and $N \Phi_{n}(f)$ we must leave our maps and homomorphisms in the uncancelled form, when dealing with iterates of standard representatives. So then, in this example, we must write $f^{2}$ as $f^{2}(a)=h(a) h(b) h(a)=$ $a b a \cdot b A \cdot a b a$ and $f^{2}(b)=h(b) h(A)=b A \cdot A B A$. In this form the important fixed points $x_{3}$ and $x_{4}$ correspond to the adjacent $A$ and $a$. To explain further, consider $x_{2}$; it lies in the part of 1 that is sent by $f$ to 3 . As we shall see later from the geometry, $f\left(x_{2}\right)=x_{4}$. That is, $x_{4}$ lies in the geometric orbit of $x_{2}$. Similarly, $f\left(x_{7}\right)=x_{3}$, and thus $x_{7}$ is in an orbit of length two. All this would be invisible if we used a standard representative of $h^{2}$. As we will see in Section 4, we make strong use of the geometric orbits to help us figure out the (algebraic) Reidemeister orbits. Thus we must keep track of all fixed points of $f^{n}$ when calculating the Nielsen periodic point numbers.

To be clear about what we mean by the unreduced form of an iterate, we note that the unreduced form for $h^{3}$ is $h^{3}(a)=a b a b A a b a \cdot b A A B A \cdot a b a b A a b a$ and $h^{3}(b)=b A A B A \cdot A B A a B A B A$. In other words, we do not cancel ever in computing all iterates up to $f^{n}$.

The problem now is simply this: The proof of Wagner's result given in [21] relies heavily on the homomorphism being given in its reduced form. So to use it for iterates, we would need to use the reduced form of the iterates $h^{n}$ of $h$. But to do this would invalidate our second technique. In particular, Wagner's proof does not consider the Nielsen equivalence of fixed points associated with canceling segments of the image words, such as $x_{3}$ and $x_{4}$ in the example above. Thus we need to extend Wagner's algorithm to ensure that it does indeed determine all Nielsen equivalences for iterates $h^{n}$ written in unreduced form. The proof of the following lemma is left to the reader. The proof of Theorem 3.3 is quite technical, and is presented in Section 7. Both are generalizations of Wagner's ideas.

$\left({ }^{3}\right)$ As an example, consider $f: S^{1} \rightarrow S^{1}$ of degree -1 . Then $f^{2}$ is homotopic to the identity on $S^{1}$, and of course $N \Phi_{2}\left(1_{S^{1}}\right)=0$. However, $N \Phi_{2}(f)=2$. 
Lemma 3.2. Let $f$ be a standard representative for $h=f_{*}: G \rightarrow G$, let $n$ be a positive integer, and let $c \in \mathcal{G}$. Suppose further that $f_{*}^{n}(c)$ is written in its unreduced form. Then there is a bijection between the set $\Phi\left(\left.f^{n}\right|_{c}\right) \backslash\left\{x_{0}\right\}$ and the occurrences of $c$ and $C$ in the words $f_{*}^{n}(c)$.

TheOREm 3.3 (Extension of Theorem 2.12). Let $f$ be a standard representative of a homomorphism $h$ with remnant. Then the proof of Theorem 2.12 extends, in a natural way, to prove that Wagner's algorithm finds all Nielsen equivalences between fixed points of any iterate of $f$.

Theorem 3.3 then essentially allows us to ignore the fact that $h^{n}$ is unreduced, and to rest assured that the Wagner tails of the unreduced form do indeed find all Nielsen equivalences of fixed points of $f^{n}$.

Our next example shows that while the Wagner tails can be helpful in determining the Reidemeister orbits and the boosting data, they do not give complete information.

EXAMPLE 3.4. Let $f$ be the standard representative for the homomorphism on the figure eight given by $f_{*}(a)=a B a$ and $f_{*}(b)=A A b$. There are four fixed points at level 1 , denoted by $x_{0}, x_{1}, x_{2}$ and $x_{3}$. We leave it to the reader to show that all four points are in the same essential Nielsen class, and that $[1]^{1}$ is the corresponding Reidemeister class. The second iterate is given by $f^{2}(a)=a B a \cdot B a a \cdot a B a$ and $f^{2}(b)=A b A \cdot A b A \cdot A A b$. In addition to the basepoint there are nine fixed points of $f^{2}$. Six of these are in the $a$ loop, and three in the $b$ loop. We use $y_{1}, \ldots, y_{9}$ to denote these in the usual order.

\begin{tabular}{crrrr}
\hline Fixed point for $f^{2}$ & loop & index & $W_{y}$ & \multicolumn{1}{c}{$\bar{W}_{y}$} \\
\hline$y_{0}=x_{0}$ & -- & +1 & 1 & 1 \\
$y_{1}$ & $a$ loop & -1 & 1 & $B a B a a a B a$ \\
$y_{2}$ & $a$ loop & -1 & $b A$ & $B a a a B a$ \\
$y_{3}$ & $a$ loop & -1 & $b A b A$ & $a a B a$ \\
$y_{4}$ & $a$ loop & -1 & $A b A b A$ & $a B a$ \\
$y_{5}$ & $a$ loop & -1 & $A A b A b A$ & $B a$ \\
$y_{6}$ & $a$ loop & -1 & $b A A A b A b A$ & 1 \\
$y_{7}$ & $b$ loop & -1 & $a$ & $A A b A A A b$ \\
$y_{8}$ & $b$ loop & -1 & $a a B a$ & $A A A b$ \\
$y_{9}$ & $b$ loop & -1 & $a a a B a a B a$ & 1 \\
\hline
\end{tabular}

Note that we have labeled the elements $W_{y}$ and $\bar{W}_{y}$. This is to emphasize the fact that the $W$ 's and the $\bar{W}$ 's are iterate specific. That is, each iterate has its own equivalence relation on $\pi_{1}(X)$.

Since $f$ has remnant, so also does $f^{2}$. By Theorem 3.3 the partition determined by the table gives the complete Nielsen relation on $\Phi\left(f^{2}\right)$. So 
there are six distinct Nielsen classes given by the partition $\left\{y_{0}, y_{1}, y_{6}, y_{9}\right\}$, $\left\{y_{2}\right\},\left\{y_{3}, y_{8}\right\},\left\{y_{4}\right\},\left\{y_{5}\right\}$ and $\left\{y_{7}\right\}$. These are represented respectively by the Reidemeister classes $[1]^{2},[b A]^{2},[a a B a]^{2},[a B a]^{2},[B a]^{2}$ and $[a]^{2}$.

This being algebraic topology, the usual way to determine Reidemeister orbits is purely algebraic. For example, it is clear that $[1]^{1}$ boosts to $[1]^{2}$, and since $f_{*}\left([1]^{2}\right)=[1]^{2}$, the orbit of $[1]^{2}$ is $\left\langle[1]^{2}\right\rangle=\left\{[1]^{2}\right\}$, that is, it consists of a single class. This orbit is essential but reducible, and does not contribute to $N_{2} P_{2}(f)$. Continuing to partition the set of Reidemeister classes into orbits, we have $f_{*}\left([a]^{2}\right)=[f(a)]^{2}=[a B a]^{2}$. Since both $a$ and $a B a$ appear in the table, we easily see $\left(^{4}\right)$ that the orbit of $[a]^{2}$ is $\left\{[a]^{2},[a B a]^{2}\right\}$, and that this orbit is irreducible. We also note that we can deduce that $f\left(\left\{y_{4}\right\}\right)=\left\{y_{7}\right\}$. To put it yet another way, $f\left(W_{y_{7}}\right)=\bar{W}_{y_{4}}$. Similarly since $f_{*}\left([B a]^{2}\right)=[B a a a B a]^{2}=$ $[b A]^{2}$, we have $\left\langle[B a]^{2}\right\rangle=\left\{[B a]^{2},[b A]^{2}\right\}$. In fact, since both of these orbits are of algebraic length 2, they are irreducible. So the Wagner tails can indeed be useful in determining orbits. But they do not always work. Consider for example the class $[a a B a]^{2}$. The table presents us with three different Reidemeister representatives of this class, $a a B a, b A b A$, and $A A A b$. Already fully canceled as presented, we see that their $f_{*}$ images are respectively

$a B a a B a B a a a B a, A A b A b A A A b A b A$ and $A b A A b A A b A A A b$, none of which appear as a Wagner tail in the table. The Wagner tails then may or may not help in the determination of orbits. In the case of $[a a B a]^{2}$ the tails simply do not help. To have the results of the example in one place, we remark that in fact we will see below, by various ad hoc methods, that $\left\langle[a a B a]^{2}\right\rangle=\left\{[a a B a]^{2}\right\}$, and that this orbit is essential. An easy search shows that $[a a B a]^{2}$ reduces to $[a]^{1}$, and abelianization techniques prove that $[a]^{1}$ is empty. We therefore have $N(f)=N \Phi_{1}(f)=N P_{1}(f)=1$, $N P_{2}(f)=2 \cdot 2=4$, and $N \Phi_{2}(f)=1+4+1=6$.

From the example, then, we see that while Wagner's (extended) algorithm determines $N\left(f^{n}\right)$ easily, we must find other techniques to complete our calculations of $N \Phi_{n}(f)$ and $N P_{n}(f)$. In particular, we must find other ways to determine the Reidemeister orbits. There are in fact a number of ad hoc techniques that can be used. In this case we can determine the orbit of $[a a B a]^{2}$ above by a number of methods. The first way is to use the geometry. By results from Section 4, we will see that $f\left(y_{3}\right)=y_{8}$. Then, using the diagram 1.14(i) in [11], and the table above, we find that

$$
\begin{aligned}
f_{*}\left([a a B a]^{2}\right) & =f_{*} \circ \varrho_{2}\left(\left\{y_{3}, y_{8}\right\}\right)=\varrho_{2} \circ f\left(\left\{y_{3}, y_{8}\right\}\right)=\varrho_{2}\left(\left\{f\left(y_{3}\right), f\left(y_{8}\right)\right\}\right) \\
& =\varrho_{2}\left(\left\{y_{8}, y_{3}\right\}\right)=[a a B a]^{2} .
\end{aligned}
$$

$\left({ }^{4}\right)$ A standard fact from periodic point theory is that the (algebraic) length of a Reidemeister orbit is less than or equal to the lowest level to which the orbit can be algebraically reduced (see also Lemma 5.3). 
Another way to see the orbit of $[a a B a]^{2}$ is to note that $\left\{y_{3}, y_{8}\right\}$ is the only Nielsen class for $f^{2}$ that contains just two points. We then use the fact that $f: \Phi\left(f^{2}\right) \rightarrow \Phi\left(f^{2}\right)$ is a bijection, so the $f$ image of this class must be taken to a class with exactly two elements. But of course $\left\{y_{3}, y_{8}\right\}$ is the only twoelement class. A third method is to recall that $f_{*}$ is index preserving. Because $\left\{y_{3}, y_{8}\right\}$ is the only class with index $-2, f_{*}$ must send $\left\{y_{3}, y_{8}\right\}$ to itself. A final ad hoc method is simply to search for solutions in the hopes of finding one. For example, to test whether $f_{*}\left([a a B a]^{2}\right)=[B a]^{2}$ in Example 3.4, we could search for a $z \in G$ satisfying $f_{*}(B a)=z a a B a f_{*}(Z)$. For how long would we search? A brute force search would never end because in this case there is no solution, as shown above. A much more sophisticated discussion of the relationship between the algebra and the geometry is in fact the subject of Section 4.

The ad hoc methods, then, can be useful at times but are by nature often inconclusive. We seek a straightforward algorithm for finding Reidemeister orbits. In the next section we show that the orbits of non-empty Reidemeister classes are determined by the geometry.

In contrast, the Wagner tails are reliably useful for determining boosts of non-empty Reidemeister classes, as stated in the following lemma. Because we do not use this result, the proof is left to the reader.

Lemma 3.5. Suppose that the fixed point $x$ of $f^{m}$ has Wagner tail $W_{x}:=$ $W_{x, m}$. Let $n=k m$ with $k>1$. Then $\iota_{m, n}\left(W_{x, m}\right)=W_{x, n}$, with $W_{x, n}$ the Wagner tail for $x$, considered as a fixed point of $f^{n}$.

4. Geometric itineraries, addresses, orbits, and reductions of points. The considerations of this section do not require that the map under consideration have remnant. So far, given an $h: G \rightarrow G$ with $G=$ $\left\langle a_{1}, \ldots, a_{r}\right\rangle$, we can produce, as in Section 2.2, a standard representative $f: \bigvee S^{1} \rightarrow \bigvee S^{1}$ of $h$ with $h=f_{*}$. As we saw there, iterates of standard representatives are not necessarily standard representatives. Using our extension of Wagner's algorithm in Section 3, we are able to determine all the Nielsen classes of each iterate and also the corresponding Reidemeister classes. In Section 3, using Example 3.4 we saw that the $W$ 's and $\bar{W}$ 's do not always completely determine the Reidemeister orbits. In this section, we develop a geometric method for identifying all non-empty Reidemeister orbits.

So far, our examples have been fairly simple. In Example 3.4, Reidemeister orbits were completely determined using ad hoc methods. One does not have to go far, however, to find that without some systematic methodology, we soon become unstuck. Before we proceed with the geometric methods of this section, we give an example that suggests how easily things can get out of hand. 
EXAMPLE 4.1. Let $f$ be a standard representative of $h$ on the figure eight, where $h(a)=a^{5}$ and $h(b)=(a b)^{2} A$. Then $f_{*}^{2}(a)=a^{25}$ and $f_{*}^{2}(b)=$ $a^{5}(a b)^{2} a^{4}(a b)^{2} A^{6}$. Below we list some important calculations for the second iterate, performed by Magma [2]. Let $k_{n}$ denotes the $k$ th fixed point of $f^{n}$, and $\mathbf{2 5}=\{1,2, \ldots, 25\}$. Also, $0_{n}$ is the wedge point regarded as a fixed point at level $n$. In the following table $u=a^{5} b a b$.

\begin{tabular}{ccccc}
\hline$\Phi\left(f^{2}\right)$ & $W$ & $\bar{W}$ & $f_{*}(W)$ & $f_{*}(\bar{W})$ \\
\hline $0_{2}$ & 1 & 1 & 1 & 1 \\
$i_{2} \forall i \in \mathbf{2 5}$ & $A^{i-1}$ & $a^{25-i}$ & $A^{5(i-1)}$ & $a^{125-5 i}$ \\
$26_{2}$ & $A^{6}$ & $a b u A^{6}$ & $A^{30}$ & $a u a^{20} u^{2} A^{31}$ \\
$27_{2}$ & $A B A^{6}$ & $u A^{6}$ & $A^{4} u^{-1} A^{26}$ & $a^{21} u^{2} A^{31}$ \\
$28_{2}$ & $A^{5} u^{-1} A$ & $a b A^{6}$ & $A^{24} u^{-2} A^{26}$ & $a u A^{31}$ \\
$29_{2}$ & $A B A^{5} u^{-1} A$ & $A^{6}$ & $A^{4} u^{-1} A^{20} u^{-2} A^{26}$ & $A^{30}$ \\
\hline
\end{tabular}

In addition, we can also look at the level 1 classes and the boosting function $\iota_{1,2}$ for this $f$. We have

\begin{tabular}{cccc}
\hline$\Phi(f)$ & $W$ & $\bar{W}$ & $\iota_{1,2}(W)$ \\
\hline $0_{1}$ & 1 & 1 & 1 \\
$i_{1}(i=1, \ldots, 5)$ & $A^{i-1}$ & $a^{5-i}$ & $A^{6(i-1)}$ \\
$6_{1}$ & $A$ & $a b A$ & $A^{6}$ \\
$7_{1}$ & $A B A$ & $A$ & $A B A^{5} B A B A^{6}$ \\
\hline
\end{tabular}

First we use Wagner's algorithm to study the orbits at level 2. Let $W$ be a Wagner tail at level 1. From Lemma 3.5, $\iota_{1,2}(W)$ is a Wagner tail at level 2, and in some cases $f_{*}(W)$ or $f_{*}(\bar{W})$ is a Wagner tail at level 2 as well. Also, $f_{*}$ preserves the index of a class. Using such ad hoc methods we can deduce the following orbits at level 2 (orbits that reduce geometrically to level 1 have a $*$ ). All of these orbits are essential.

$$
\begin{array}{cccc}
\left\langle\left\{0_{2}, 1_{2}, 25_{2}\right\}^{*}\right\rangle, & \left\langle\left\{7_{2}, 26_{2}, 29_{2}\right\}^{*}\right\rangle, & \left\langle\left\{2_{2}\right\},\left\{6_{2}\right\}\right\rangle, & \left\langle\left\{3_{2}\right\},\left\{11_{2}\right\}\right\rangle, \\
\left\langle\left\{4_{2}\right\},\left\{16_{2}\right\}\right\rangle, & \left\langle\left\{5_{2}\right\},\left\{21_{2}\right\}\right\rangle, & \left\langle\left\{10_{2}\right\},\left\{22_{2}\right\}\right\rangle, & \left\langle\left\{13_{2}\right\}^{*}\right\rangle, \\
\left\langle\left\{15_{2}\right\},\left\{23_{2}\right\}\right\rangle, & \left\langle\left\{19_{2}\right\}^{*}\right\rangle, & \left\langle\left\{20_{2}\right\},\left\{24_{2}\right\}\right\rangle &
\end{array}
$$

The ad hoc methods did not determine the Nielsen orbits of the Nielsen classes $\{8\},\{9\},\{12\},\{14\},\{17\},\{18\},\{27\}$, and $\{28\}$, nor whether each orbit reduces. We continue this example at the end of Section 4 , after developing some new geometric techniques.

To see how the geometry can help, we return for a moment to Example 3.1. In that example we saw that $f\left(x_{3}\right)$ lies in the $b$ loop. Note that this immediately rules out a number of possibilities for the image under $f_{*}$ of the Reidemeister class containing $x_{3}$. In particular, it rules out any class 
whose intersection with the $b$ loop is empty. In fact, we can do very much better than this rough approximation. For each $n$, we will assign an $n$ level address to each periodic point. These addresses are made longer and more precise at each new iterate, as in symbolic dynamics. The scheme we develop for labeling the said locations allows us to determine not only which points (and hence ultimately which Nielsen classes) are in the same geometric orbit, but also which points are fixed points of lower iterates. We begin by defining the concept of an adjacency matrix.

Definition 4.2. Let $G=\pi_{1}(X)=\left\langle g_{1}, \ldots, g_{l}\right\rangle$, and let $h: G \rightarrow G$. We let $k:=\sum_{i=1}^{l}\left|h\left(g_{i}\right)\right|$ and let $f$ be the standard representative of $h$. Define the $k \times k$ matrix $Q=\left(q_{i, j}\right)$, with $q_{i, j} \in\{0,1\}$, determined by the condition $q_{i, j}=1$ if and only if $j \subset f(i)$. Here $i$ and $j$ are pieces of the circles of $X$ as defined in Section 2.2. The matrix $Q$ is called the adjacency matrix of $f$.

It is not hard to see that $f(i) \cap j$ is either empty or is a connected segment of $X$. Our next step is to use the adjacency matrix to define legitimate itineraries. This may remind the reader of non-zero cycles in the computation of determinants.

Definition 4.3. Let $n$ be a fixed positive integer. A legitimate itinerary at level $n$ is a vector $\vec{r}=\left(r_{1}, \ldots, r_{n+1}\right)$ where $r_{i} \in\{1, \ldots, n+1\}$ is such that $q_{r_{i}, r_{i+1}}=1$. A legitimate itinerary $\vec{r}=\left(r_{1}, \ldots, r_{n+1}\right)$ in which $r_{1}=r_{n+1}$ is called a round trip.

ExAmPle 2.9 (part four). Consider the map $f$ of the figure eight given by $f_{*}(a)=a a b$ and $f_{*}(b)=B a$. Then the adjacency matrix $Q$ of $f$ is

$$
Q=\left(\begin{array}{lllll}
1 & 1 & 1 & 0 & 0 \\
1 & 1 & 1 & 0 & 0 \\
0 & 0 & 0 & 1 & 1 \\
0 & 0 & 0 & 1 & 1 \\
1 & 1 & 1 & 0 & 0
\end{array}\right)
$$

So for example $q_{4,4}=1$, because the fourth part of $X$, namely the first part of the $b$ loop, is taken by $f$ onto the $b$ loop. Notice that the restriction of $f$ to the open interval 4 is a linear homeomorphism of the $b$ loop. By the fixed point property (or otherwise) the interval 4 must contain exactly one fixed point by construction.

Fix $n$ to be 1 . Then a legitimate itinerary at level 1 is a vector $\left(r_{1}, r_{2}\right)$ with $q_{r_{1}, r_{2}}=1$. Notice that $(1,1),(1,2)$, and $(2,1)$ are legitimate itineraries but $(4,1)$ is not.

Now fix $n$ to be 2. A legitimate itinerary at this level is a triple $\left(r_{1}, r_{2}, r_{3}\right)$ with $q_{r_{i}, r_{(i+1)}}=1$. So for example the triples $(1,2,1),(4,4,4)$, and $(5,3,5)$ are 
legitimate itineraries, since $q_{1,2}=q_{2,1}=1, q_{4,4}=1$, and also $q_{5,3}=q_{3,5}=1$. On the other hand, $(1,3,1)$ is not a legitimate itinerary. As we will see, the itineraries $(1,2,1)$ and $(5,3,5)$ correspond to 2-periodic points of least period 2, while $(4,4,4)$ corresponds to a fixed point boosting from level 1 .

Each legitimate itinerary $\vec{r}=\left(r_{1}, \ldots, r_{n+1}\right)$ uniquely defines a segment $r_{1} \cdots r_{n+1}$ of $r_{1}$, which can be defined inductively. Starting at level 1 if $\left(r_{1}, r_{2}\right)$ is a legitimate itinerary, then $r_{1} r_{2}$ is that part of $r_{1}$ that is taken by $f$ homeomorphically onto $r_{2}$. We assume that all segments $r_{1} \cdots r_{n}$ associated with legitimate itineraries $\left(r_{1}, \ldots, r_{n}\right)$ at level $n-1$ have been defined. Next, let $\left(r_{1}, \ldots, r_{n+1}\right)$ be a legitimate itinerary at level $n$. We define $r_{1} \cdots r_{n+1}$ to be that part of $r_{1} \cdots r_{n}$ that is taken homeomorphically by $f^{n}$ onto $r_{n+1}$. We can put this into other words by saying that

$$
r_{1} \cdots r_{n+1}=\left(f^{n}\right)^{-1}\left(r_{n}\right) \cap r_{1} \cdots r_{n} .
$$

The segments just defined have the following interesting properties.

Lemma 4.4. Let $\vec{r}=\left(r_{1}, \ldots, r_{n+1}\right)$ be a legitimate itinerary at level $n$. Then

(i) $r_{1} \cdots r_{n} \subset r_{1} \cdots r_{n-1}$, and in particular $r_{1} \cdots r_{n} \subset r_{1}$.

(ii) $f\left(r_{1} \cdots r_{k}\right)=r_{k+1}$ for each $k=2, \ldots, n$.

The jist of what we do now is, for each $n$, to associate a unique " $n$-level address" with every legitimate round trip at level $n$. We then show that every such address is the "home" of a unique fixed point of $\Phi\left(f^{n}\right)$. In this way there are one-to-one correspondences between $n$-level round trips, $n$ level addresses and fixed points of $f^{n}$. To start this process, we claim (see 4.6) that every round trip $\vec{r}=\left(r_{1}, \ldots, r_{n}, r_{1}\right)$ at level $n$ is uniquely associated with one fixed point of $f^{n}$. In order to define this correspondence, notice that by applying the definition of a round trip inductively we must have $f^{n}\left(r_{1}\right) \cap r_{1} \neq \emptyset$. The fixed point property of line segments now gives a fixed point for our correspondence. Obviously any such $n$-periodic point is contained in $r_{1}$, but we want to be far more precise about its location (its address).

Definition 4.5. Let $n \geq 1$, and let $\vec{r}=\left(r_{1}, \ldots, r_{n}, r_{1}\right)$ be a round trip at level $n$. Then we say that the segment $r_{1} \cdots r_{n}$ is the address of the round trip $\vec{r}$. We call $r_{1} \cdots r_{n}$ the level $n$ address associated with the round trip $\vec{r}=\left(r_{1}, \ldots, r_{n+1}\right)$.

Note that addresses are only defined for round trips, and that the last term of the round trip does not appear in the address (unlike in segments). At the risk of being repetitive we emphasize that if $r_{1} \cdots r_{n}$ is the address of a round trip, then $f^{n}$ takes the segment which is denoted $r_{1} \cdots r_{n}$ homeomorphically onto $r_{n+1}=r_{1}$. In other words, $r_{1} \cdots r_{n+1}=\left(f^{n}\right)^{-1}\left(r_{n}\right) \cap r_{1} \cdots r_{n}$. This is straightforward when $f$ preserves the orientation of a given segment. However, care must be taken when upper case letters are involved. 
To illustrate this, we look at an example. In 2.9 the segment 2 is taken onto the $a$ loop with the same orientation. So 21 is the first part of 2,22 is the second part of 2 , and 23 is the third part. However, things are not always this simple. Consider the $b$ loop in this same example. Because the segment 4 is sent to the $b$ loop with orientation reversed, the first piece of 4 is the segment 45 , while the second piece of 4 is 44 as shown in the diagram.

Note that we are ignoring the neighborhood $N$ in our picture and that the dashed line represents a partial diagonal, which of course shows the existence of a fixed point in 44 .

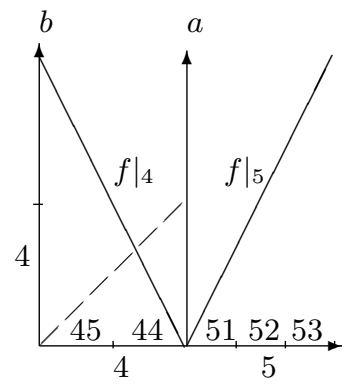

The point of course is that it is the second half of 4 that is taken by $f$ onto 4. A perhaps helpful way to understand addresses of periodic points is to think of "houses" numbered backwards down "streets" that are attached to upper case letters.

Next we state the crucial connection between fixed points of iterates and addresses.

LEMma 4.6. There is a one-to-one correspondence between the set of distinct round trips at level $n$ and the set $\Phi\left(f^{n}\right) \backslash\left\{x_{0}\right\}$. Moreover, the unique fixed point associated with a round trip $\left(r_{1}, \ldots, r_{n}, r_{1}\right)$ resides at the address $r_{1} \cdots r_{n}$.

Proof. Let $\vec{r}=\left(r_{1}, \ldots, r_{n}, r_{1}\right)$ be a round trip. By the inductive definition of segment, we know that $f^{n}$ takes $r_{1} \cdots r_{n}$ homeomorphically onto $r_{1}$. Since $r_{1} \cdots r_{n} \subset r_{1}$, there is a unique fixed point of $f^{n}$ at the address $r_{1} \cdots r_{n}$. Clearly this fixed point is not $x_{0}$. Now let $x \in \Phi\left(f^{n}\right) \backslash\left\{x_{0}\right\}$. Let $r_{1}$ be the segment containing $x, r_{2}$ be the segment containing $f(x)$, and $r_{i}$ be the segment containing $f^{i-1}(x)$. Clearly, since $f^{n}(x)=x$, we have $r_{n+1}=r_{1}$. This determines a vector $\left(r_{1}, \ldots, r_{n}, r_{1}\right)$. It remains to show that $q_{i, i+1}=1$. If $y=f^{i}(x)$, then we see that $y \in r_{i}$ and $f(y) \in r_{i+1}$. So clearly $f\left(r_{i}\right) \cap r_{i+1} \neq \emptyset$. It should be clear that the composition of these two assignments is the identity on the respective sets.

The uniqueness of the assignment, fixed point to address, easily gives the following results concerning orbits and boosting. 
LEMMA 4.7. If $\vec{r}=\left(r_{1}, \ldots, r_{n}, r_{1}\right)$ is a round trip, and if $x \in r_{1} \cdots r_{n}$ is the corresponding fixed point of $f^{n}$, then the fixed point $f(x)$ of $f^{n}$ lies in $r_{2} \cdots r_{n} r_{1}$. Moreover, if $r_{1} \cdots r_{m}$ is the address of a periodic point of period $m$ at level $m$, then its address at level $p m$ is $r_{1} \cdots r_{m} \cdots \cdots r_{1} \cdots r_{m}$, where the cycle $r_{1} \cdots r_{m}$ is repeated $p$ times.

For convenience, we now identify each $x \in \Phi\left(f^{n}\right) \backslash\left\{x_{0}\right\}$ with its $n$-level address, which we denote by $r_{1} \cdots r_{n}$. The $n$-level address for $x_{0}$ is defined to be $00 \cdots 0$. We define a function $f: \Phi\left(f^{n}\right) \rightarrow \Phi\left(f^{n}\right)$ by $f\left(r_{1} r_{2} \cdots r_{n}\right)=$ $r_{2} \cdots r_{n} r_{1}$. The next result essentially says that the geometric orbit of a fixed point $x$ of $f^{n}$ can be determined by its address.

Lemma 4.8 (Geometric orbits of points). Suppose that $x \in \Phi\left(f^{n}\right)$ has address $r=r_{1} r_{2} \cdots r_{n}$. Then the geometric orbit $\langle x\rangle$ of $x$ is the set $\{r, f(r)$, $\left.f^{2}(r), \ldots, f^{n-1}(r)\right\}$.

Note that this set might contain fewer than $n$ distinct elements.

Lemma 4.9 (Geometric reductions of points). Let $x=r_{1} \cdots r_{n}$ be $a$ fixed point of $f^{n}$. Suppose that $d$ is a divisor of $n$ and that $r_{i}=r_{j}$ whenever $i \equiv j \bmod d$. Then $x$ is also a fixed point of $f^{d}$ and as such $x=r_{1} \cdots r_{d}$. The minimum period of $x$ is the minimum such divisor $d$ of $n$.

EXAMPLE 4.1 (part two). By applying our addressing system to the periodic points of Example 4.1, we are now able to gives the complete list of orbits at level two. Each orbit of Nielsen classes is presented in two forms in the table below. For example, the Nielsen class of the fixed point $13_{2}$ is a singleton, $\left\{13_{2}\right\}$. The fixed point has address 33, so $\left\langle\left\{13_{2}\right\}\right\rangle=\langle\{33\}\rangle$.

\begin{tabular}{cc}
\hline Orbit of $\mathbf{C}^{2}$ & Address form \\
\hline$\left\langle\left\{0_{2}, 1_{2}, 25_{2}\right\}\right\rangle$ & $\langle\{00,11,55\}\rangle$ \\
$\left\langle\left\{7_{2}, 26_{2}, 29_{2}\right\}\right\rangle$ & $\langle\{22,77,99\}\rangle$ \\
$\left\langle\left\{2_{2}\right\}\right\rangle=\left\langle\left\{6_{2}\right\}\right\rangle$ & $\langle\{12\}\rangle=\langle\{21\}\rangle$ \\
$\left\langle\left\{3_{2}\right\}\right\rangle=\left\langle\left\{11_{2}\right\}\right\rangle$ & $\langle\{13\}\rangle=\langle\{31\}\rangle$ \\
$\left\langle\left\{4_{2}\right\}\right\rangle=\left\langle\left\{16_{2}\right\}\right\rangle$ & $\langle\{14\}\rangle=\langle\{41\}\rangle$ \\
$\left\langle\left\{5_{2}\right\}\right\rangle=\left\langle\left\{21_{2}\right\}\right\rangle$ & $\langle\{15\}\rangle=\langle\{51\}\rangle$ \\
$\left\langle\left\{10_{2}\right\}\right\rangle=\left\langle\left\{22_{2}\right\}\right\rangle$ & $\langle\{25\}\rangle=\langle\{52\}\rangle$ \\
$\left\langle\left\{13_{2}\right\}\right\rangle$ & $\langle\{33\}\rangle$ \\
$\left\langle\left\{15_{2}\right\}\right\rangle=\left\langle\left\{23_{2}\right\}\right\rangle$ & $\langle\{35\}\rangle=\langle\{53\}\rangle$ \\
$\left\langle\left\{19_{2}\right\}\right\rangle$ & $\langle\{44\}\rangle$ \\
$\left\langle\left\{20_{2}\right\}\right\rangle=\left\langle\left\{24_{2}\right\}\right\rangle$ & $\langle\{45\}\rangle=\langle\{54\}\rangle$ \\
$\left\langle\left\{8_{2}\right\}\right\rangle=\left\langle\left\{12_{2}\right\}\right\rangle$ & $\langle\{23\}\rangle=\langle\{32\}\rangle$ \\
$\left\langle\left\{9_{2}\right\}\right\rangle=\left\langle\left\{17_{2}\right\}\right\rangle$ & $\langle\{24\}\rangle=\langle\{42\}\rangle$ \\
$\left\langle\left\{14_{2}\right\}\right\rangle=\left\langle\left\{18_{2}\right\}\right\rangle$ & $\langle\{34\}\rangle=\langle\{43\}\rangle$ \\
$\left\langle\left\{27_{2}\right\}\right\rangle=\left\langle\left\{28_{2}\right\}\right\rangle$ & $\langle\{79\}\rangle=\langle\{97\}\rangle$ \\
\hline
\end{tabular}


Note the ease with which orbits and reducibility can be determined using the addresses. The last four lines are orbits that we could not compute without these new techniques.

All these orbits are essential. A routine calculation exhibits eleven irreducible orbits at level 2, and four reducible orbits at level 2. The latter reduce to four distinct essential orbits (i.e., classes) at level 1. Thus

$$
N P_{2}(f)=2 \times 11=22 \quad \text { and } \quad N \Phi_{2}(f)=22+4=26 .
$$

5. Empty reductions: word length arguments. One of the things that makes computation of the Nielsen periodic numbers complex is the possibility that an orbit of geometric length $n$ but algebraic length less than $n$ may be reducible to an empty orbit. This occurs exactly when the algebraic length is strictly less than the geometric depth of the orbit. We explain this in more detail below.

As can be seen from their addresses, all orbits of length 1 in Example 4.1 at level 2 have non-empty reductions to level 1 . However, in general a Reidemeister orbit can reduce to another Reidemeister orbit that does not represent any fixed points. Such an orbit is called an empty Reidemeister orbit. We had an example of this already in Example 3.4, where $\left\langle[a a B a]^{2}\right\rangle$, which is of length 1 at level 2, reduced to the empty orbit $\left\langle[a]^{1}\right\rangle$ at level 1 . Whether such orbits reduce or not affects the values of $N P_{n}(f)$ and $N \Phi_{n}(f)$. To explain what is happening here, recall the fundamental diagram 1.14(ii) from [11]:

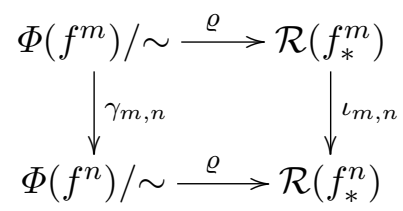

See Section 2 for definitions of $\Phi\left(f^{m}\right) / \sim$ and $\gamma_{m, n}$. Because the functions $\varrho$ are not surjective, there might be a class $\mathbf{C}^{n} \in \Phi\left(f^{n}\right) / \sim$ for which there is a Reidemeister class $[\alpha]^{m} \in \mathcal{R}\left(f_{*}^{m}\right)$ with $\iota_{m, n}\left([\alpha]^{m}\right)=\varrho\left(\mathbf{C}^{n}\right)$, but with $[\alpha]^{m}$ not in the image of $\varrho: \Phi\left(f^{m}\right) / \sim \rightarrow \mathcal{R}\left(f_{*}^{m}\right)$. By definition, such an $[\alpha]^{m}$ is an empty Reidemeister class.

Now let $m=1$ and $n=2$. As remarked above, Example 3.4 exhibits this with $\varrho\left(\mathbf{C}^{2}\right)=\varrho\left(\left\{y_{3}, y_{8}\right\}\right)=[a a B a]^{2}$ and with $\alpha=[a]^{1}$. Clearly the class $\left\{y_{3}, y_{8}\right\}$ is not in the image of $\gamma_{1,2}$. However, geometric depth is not a homotopy invariant, and so this certainly does not preclude $[a a B a]^{2}$ being in the image of $\iota_{1,2}$. If the Reidemeister class were indeed reducible, and if in addition $N P_{2}(f)$ were sharp, this would represent the fact that $f$ would 
be homotopic to a map that coalesced the two periodic points $y_{3}$ and $y_{8}$ of $f$ into a single fixed point $\left({ }^{5}\right)$ at level 1.

REMARK 5.1 (The remaining question for $N P_{n}(f)$ ). Given $[\beta]^{m}$, an essential class at level $m$ with $m \mid n$ that contains only periodic points of minimal period $m$, does there exist an $[\alpha]^{k}$ at level $k$ to which $[\beta]^{m}$ reduces? Of necessity, $[\alpha]^{k}$ will be empty, and hence inessential. In particular, we must ask: for which divisors $k$ of $m$ must we search for solutions? Our solution when this happens is to seek a bound on the length of possible solutions $\alpha$ that must be tested in the equation (cf. Fact 2.2).

EXAMPLE 5.2. The following example, which exhibits algebraic length less than depth for an essential orbit, is adapted from Example 3 from [8].

Let $G=\langle a, b\rangle$, and let $f$ be such that $f_{*}(a)=B^{2}$ and $f_{*}(b)=B A$. Then $f_{*}^{2}(a)=a b a b$ and $f_{*}^{2}(b)=a b^{3}$. Let $x$ be the fixed point of $f^{2}$ with address 24. Then the geometric orbit of $x$ has length 2. But by Wagner's algorithm we see that the corresponding algebraic orbit has length 1 because $\bar{W}_{x}=\bar{W}_{f(x)}$. This algebraic orbit is $\left\langle[B A]^{2}\right\rangle$. The example from [8], upon which this one is based, used abelianization to show that there is no solution to the 4-generator version of $\alpha f_{*}(\alpha)=B A$. We leave it to the reader to deduce that in this case as well there is no solution. Thus this algebraic orbit is irreducible, with length 1 and depth 2 .

We start with the following lemma, which restricts significantly the amount of work needed to determine whether a class $[\beta]^{n}$ is reducible. The first part is standard periodic point theory (in fact, we have already used it).

Lemma 5.3. If the orbit of $[\beta]^{n} \in \mathcal{R}\left(f_{*}^{n}\right)$ has (algebraic) length $n$, then the orbit is irreducible. If the orbit has length $l<n$, then $l \mid n$, and then $[\beta]^{n}$ is reducible if and only if it is reducible to some level $n / p$, where $p$ is a prime dividing $n / l$.

This lemma can provide a huge improvement in efficiency. There are two reasons why this is so. Firstly, suppose for example that we are working at level $n=36$, and we have an orbit $\left\langle[\beta]^{36}\right\rangle$ of algebraic length 18 . Then, since $36 / 18=2$, by the lemma $\left\langle[\beta]^{36}\right\rangle$ is reducible if and only if it is reducible to some orbit $\left\langle[\alpha]^{18}\right\rangle$ at level 18. So then, for this particular orbit, there is just one level we need to check for reduction (as opposed to all divisors of $36)$. But there is a second advantage to the lemma. Notice first that the orbit $\left\langle[\beta]^{36}\right\rangle$ is reducible to $\left\langle[\alpha]^{18}\right\rangle$ if and only if for some representative $\beta$ of $\left\langle[\beta]^{36}\right\rangle$, there is a representative $\alpha$ of $\left\langle[\alpha]^{18}\right\rangle$ with $\iota_{18,36}(\alpha)=\beta$. Now $\iota_{18,36}(\alpha)=\alpha f_{*}^{18}(\alpha)$, so this is like working with the map $f^{18}$ going from

$\left({ }^{5}\right)$ We visualize this as, roughly, the two 2-periodic points $e^{ \pm i \theta}$ in $S^{1}$ under the flip map being coalesced into $e^{i 0}$ as $\theta$ approaches 0 . 
level 1 to level 2. The advantage of this is that the remnant of $f^{18}$ can be huge, even when the remnant of $f$ is small. In Example 4.1 the remnant grows exponentially. A longer remnant provides a more restrictive bound on the length of $\alpha$ if $\alpha$ is to boost to $\beta$ (see Theorem 5.4).

Proof of Lemma 5.3. Let $l$ be the length of the orbit $\left\langle[\beta]^{n}\right\rangle$, and let $d$ be the lowest value for which $[\beta]^{n}$ reduces to level $d$. By a standard fact of periodic point theory [11], $l \mid d$, and we know that $d \mid n$. Thus if $l=n$ we know that the orbit is irreducible. Now suppose that $l<n$. If $[\beta]^{n}$ is reducible, then $d<n$ and there is a class $[\eta]^{d}$ such that $\iota_{d, n}(\eta)=\beta$. Let $p$ be a prime divisor of $n / d$. Then $n / p$ is a multiple of $d$. Let $\tau=\iota_{d, n / p}(\eta)$. Using the properties of the boosting function, we find that

$$
\beta=\iota_{d, n}(\eta)=\iota_{n / p, n}\left(\iota_{d, n / p}(\eta)\right)=\iota_{n / p, n}(\tau) .
$$

Thus the orbit of $[\beta]^{n}$ is reducible to level $n / p$. Note also that $n / d \mid n / l$, so $p$ is a prime factor of $n / l$. The converse is immediate.

Let $g=f_{*}^{m}$ with the words $g\left(a_{i}\right)$ written in reduced form. Let $M R(m)=$ $\min \left\{\left|R_{i}\right|: R_{i}\right.$ is the remnant of $\left.g\left(a_{i}\right)\right\}$, the minimum length of the remnants $R_{i}$ of $g\left(a_{i}\right)$.

THEOREM 5.4. Let $f$ be a map with remnant.

(1) For any $\delta \in G, \delta \neq 1$, we have $\left|f_{*}^{m}(\delta)\right| \geq(M R(1))^{m}|\delta|$.

(2) If $M R(m) \geq 2$ and if $\iota_{m, n}(\alpha)=\beta$ for some $\alpha, \beta \in G$, then

$$
|\alpha| \leq \frac{1}{\mu(m, n)}|\beta|, \quad \text { where } \mu(m, n)=(M R(m))^{n / m-1}-\sum_{i=0}^{n / m-2}(M R(m))^{i} .
$$

(3) If $M R(1) \geq 2$, then $\mu(m, n) \geq 1$ for all $m$. In particular, $|\alpha| \leq|\beta|$ for all $m$.

EXAMPle 5.5. Consider the endomorphism $f_{*}(a)=a b^{3} a B^{2}=a \cdot \underline{b^{3} a B^{2}}$ and $f_{*}(b)=B A B A^{4}=\underline{B A B A^{3}} \cdot A$, with remnant underlined.

We consider the implications of Theorem 5.4 for fixed points of $f^{3}$. We want to determine whether a non-empty Reidemeister class $[\beta]^{3}$ reduces to a class $[\alpha]^{1}$. We have $M R(1)=6$, so $\mu(1,3)=29$. Thus we know that $|\alpha| \leq|\beta| / 29$. In other words, given any $\beta$ at level three, to test for a reduction to level 1 we need only consider words $\alpha$ of length less than $1 / 29$ of the length of $\beta$. However, we can also limit the lengths of the $\beta$ 's that we need to consider.

Let $[\beta]^{3}$ be an essential non-empty class that contains no fixed points of $f$. Only for such $\beta$ do we need to check for reductions to level 1. Firstly, for each such $[\beta]^{3}$, there is a point $x \in \Phi\left(f^{3}\right)$ for which $\left[W_{x}\right]^{3}=\left[\bar{W}_{x}\right]^{3}=[\beta]^{3}$. So without loss of generality, we can assume that $\beta$ is the shorter of $W_{x}, \bar{W}_{x}$, $W_{f(x)}, \bar{W}_{f(x)}, W_{f^{2}(x)}$ and $\bar{W}_{f^{2}(x)}$. Thus $|\beta| \leq \max \left\{\left(\left|f_{*}^{3}(a)\right|+1\right) / 2,\left(\left|f_{*}^{3}(b)\right|+\right.\right.$ 
1) $/ 2\}$. By a calculation done by Magma and checked by hand, $\left|f_{*}^{3}(a)\right|=309$ and $\left|\left(f_{*}\right)^{3}(b)\right|=315$. Thus the longest $\beta$ that we ever need to consider has length less than 158. Even for a $\beta$ this long, the longest $\alpha$ that we ever need to consider has at most five letters. Secondly, we can select the shortest $\beta$ from the entire orbit, and since the point $x$ has least period 3 , it is likely that $f$ takes one of $\left\{x, f(x), f^{2}(x)\right\}$ some distance from the center of $f_{*}(a)$ or $f_{*}(b)$, where either the associated $W$ or $\bar{W}$ is shorter. Thus we can quickly determine whether there are non-empty Reidemeister classes for $f^{3}$ that reduce to a Reidemeister class (empty or non-empty) for $f$.

REMARK 5.6 (The power of the bounds). Note from (3) that if we have remnant at least 2 , then we need never search for solutions for words longer than the shortest Wagner tail equivalent to $\beta$. We can, however be even more efficient in several ways. Firstly, in any given situation, we can always choose to work the algorithm with the shorter of $W_{x}$ and $\bar{W}_{x}$. Secondly, it will quickly become apparent that for very many cases the only reducible points will be in the middle sections of the loops. To see this, note that if $M R(m)$ and and $n / m$ are sufficiently large, and $|\beta|$ is sufficiently small, the $\alpha$ in (3) must be such that $|\alpha|<1$. So in these circumstances $[\beta]^{n}$ will be automatically irreducible. More precisely, apart from $[1]^{n}$, the essential Reidemeister classes at level $n$ are of the form $\left[W_{x}\right]^{n}$ for some $x \in \Phi\left(f^{n}\right) \backslash\left\{x_{0}\right\}$. For such $x, W_{x}^{-1}$ is an initial segment of an (unreduced) word $f_{*}^{n}\left(a_{i}\right)$, so that if $W_{x}$ is a long word, then $\bar{W}_{x}$ is relatively short. Since $\left[W_{x}\right]^{n}=\left[\bar{W}_{x}\right]^{n}$ we could, in such cases, put $\beta=\bar{W}_{x}$ and often deduce that many such classes are irreducible. In particular, when $\bar{W}_{x}$ is so short that our bound on $|\alpha|$ is less than one, then we know that $\bar{W}_{x}$ cannot reduce to level $m$ and so neither can $W_{x}=\beta$. So if $|\alpha|<1$, the only essential classes $W_{x}$ that can reduce to level $m$ represent fixed points $x$ from the middle of a loop of $X$ rather than from the ends.

Finally, we remark that the process involved in these word length arguments is more effective than abelianization in that it gives more information. In the example above, abelianization provides only the following: If the abelianization $\bar{\beta}$ of $\beta$ is written as $\bar{\beta}=\bar{a}^{i} \bar{b}^{j}$, then if $\beta$ has a reduction $\alpha$ we must have $|\bar{\alpha}| \leq 7|i|+3|j|$. Even if a solution $\bar{\alpha}$ to the abelianized equation is found, this is inconclusive. We still would not know whether a solution $\alpha$ exists to the equation in the fundamental group.

Proof of Theorem 5.4. Let $k=M R(1)$ for $f_{*}$. Then because $f$ has remnant we know that $\left|f_{*}(\delta)\right| \geq k|\delta|$. By induction we have $\left|f_{*}^{m}(\delta)\right| \geq$ $\left|f_{*}\left(f_{*}^{m-1}(\delta)\right)\right| \geq k\left|f_{*}^{m-1}(\delta)\right| \geq k^{m}|\delta|$.

Next we note that without loss of generality we can assume that $m=1$. (Otherwise replace $f_{*}^{m}$ with $h_{*}$.) We prove inductively that $\left|\iota_{1, n}(\alpha)\right| \geq$ $\left(k^{n-1}-\sum_{i=0}^{n-2} k^{i}\right)|\alpha|$. 
First for $n=2$ we have $\left|\iota_{1,2}(\alpha)\right|=\left|\alpha f_{*}(\alpha)\right| \geq\left|f_{*}(\alpha)\right|-|\alpha| \geq k|\alpha|-|\alpha|=$ $(k-1)|\alpha|$. Next, we use induction to get

$$
\begin{aligned}
\left|\iota_{1, n}(\alpha)\right| & =\left|\alpha f_{*}(\alpha) f_{*}^{2}(\alpha) \cdots f_{*}^{n-1}(\alpha)\right| \geq\left|f_{*}(\alpha) f_{*}^{2}(\alpha) \cdots f_{*}^{n-1}(\alpha)\right|-|\alpha| \\
& \geq\left|f_{*}\left(\alpha f_{*}(\alpha) \cdots f_{*}^{n-2}(\alpha)\right)\right|-|\alpha| \geq k\left|\alpha f_{*}(\alpha) \cdots f_{*}^{n-2}(\alpha)\right|-|\alpha| \\
& \geq k\left(k^{n-2}-\sum_{i=0}^{n-3} k^{i}\right)|\alpha|-|\alpha|=\left(k^{n-1}-\sum_{i=0}^{n-2} k^{i}\right)|\alpha| .
\end{aligned}
$$

6. An algorithm for $N P_{n}(f)$. In this section, we give an algorithm for finding $N P_{n}(f)$. As above, we have a free group $G=\pi_{1}(X)=\left\langle a_{1}, \ldots, a_{r}\right\rangle$ with generators $\mathcal{G}=\left\{a_{1}, \ldots, a_{r}\right\}$.

Algorithm 6.1 (Algorithm for finding $N P_{n}(f)$ ). Let $g: Y \rightarrow Y$ be a map of a surface with boundary or a bouquet of circles. Fix $n \in \mathbb{N}$. Then the following procedure is algorithmic.

1. Let $h=g_{*}: \pi_{1}(Y) \rightarrow \pi_{1}(Y)$ be the induced homomorphism. We require that $h$ be in its reduced form and have minimum remnant length at least 2.

2. Form the standard representative $f: X \rightarrow X$ of $h$ on the appropriate bouquet of $r$ circles $X$ and list the fixed points of $f^{n}$.

3. Determine the address, and a shortest Wagner tail, of each fixed point of $f^{n}$. (The base point has the null address and both Wagner tails are equal to 1.)

4. Determine all non-empty Reidemeister classes of $f^{n}$, using Wagner's algorithm on the Wagner tails. Disregard all inessential classes and, using the addresses, disregard all those classes that represent fixed points of lower iterates.

5. Partition the remaining non-empty Reidemeister classes into Reidemeister orbits, using the addresses and taking note of the length of each orbit. Call the quotient set $\mathcal{E O}(n)$. Partition this set of orbits into two subsets. Let $\mathcal{L}(n)$ consist of the orbits of algebraic length exactly $n$, and let $\mathcal{L}(<n)$ be the other orbits. Thus $\mathcal{E O}(n)=$ $\mathcal{L}(n) \cup \mathcal{L}(<n)$. By Lemma 5.3 all orbits in $\mathcal{L}(n)$ are irreducible.

6. Determine the subset $\mathcal{I} \mathcal{L}(<n)$ of $\mathcal{L}(<n)$ which consists of irreducible orbits of length less than $n$ as follows. Factor $n$ into primes, $n=$ $p_{1}^{r_{1}} \cdots p_{k}^{r_{k}}$. By Lemma 5.3, $\left\langle[\beta]^{n}\right\rangle$ is reducible if and only if it is reducible to level $t_{i}:=n / p_{i}$ for some $i=1, \ldots, k$. We must use our techniques to check reduction to each such level, and we do this as follows. For each orbit $\left\langle[\beta]^{n}\right\rangle \in \mathcal{L}(<n)$ select the shortest Wagner tail $W_{\left\langle[\beta]^{n}\right\rangle}$ among all representatives $\beta$ of $\left\langle[\beta]^{n}\right\rangle$. For each $i$, program the computer to search for all solutions $\alpha$ to the equation $\iota_{t_{i}, n}(\alpha)=W_{\left\langle[\beta]^{n}\right\rangle}$ 
which are of length less than or equal to the bound specified by Theorem 5.4. By Theorem 5.4, this is a finite search. If no solution is found, then $\left\langle[\beta]^{n}\right\rangle$ is not reducible to level $t_{i}$. If no solution is found for any $i=1, \ldots, k$, then $\left\langle[\beta]^{n}\right\rangle \in \mathcal{I} \mathcal{L}(<n)$.

7. The number $N P_{n}(f)$ is $n \cdot(\#(\mathcal{L}(n))+\#(\mathcal{I} \mathcal{L}(<n)))$.

REMARK 6.2. We note that it is only in Step 6 of Algorithm 6.1 that we need minimum remnant length at least two. For the other steps, we require only that the map have remnant.

The proof that Algorithm 6.1 is indeed an algorithm for computing $N P_{n}(f)$ is simple and is left to the reader. The efficiency of calculating $N P_{n}(f)$ is discussed in Remark 5.6.

As indicated earlier, any algorithm for $N \Phi_{n}(f)$ in the general case is more subtle. Actually, there are times when the simple equation $N \Phi_{n}(f)=$ $\sum_{m \mid n} N P_{m}(f)$ holds. In such cases we simply need to do multiple versions of Algorithm 6.1 (i.e. for all $m \mid n$ ). However, there are times when $N \Phi_{n}(f)>$ $\sum_{m \mid n} N P_{m}(f)$, and this case entails a whole host of new word problems that need to be solved. $N \Phi_{n}(f)$ will appear in [7].

7. Appendix: Wagner's algorithm for iterates. In this appendix we prove Theorem 3.3, which extends Wagner's Theorem (2.12). This allows us to apply Wagner's algorithm, using Wagner tails, to determine all nonempty classes (and their indices) for iterates of a standard representative $f$, even when the induced homomorphism is in unreduced form.

We assume some familiarity with the proof of Theorem 2.12 (see either Wagner's paper [21] or [5], where the proof is repeated with simplified notation and additional motivation). The only stumbling block to the generalization of Theorem 2.12 is its heavy dependence on the lemma below from [21].

Lemma 7.1 (Wagner). Let $f$ be a standard representative for a homomorphism $h: G \rightarrow G$ with remnant. Let $\alpha \in G$, and let $g, s \in \mathcal{G}^{ \pm}$. If $\alpha$ is an initial segment of $f_{*}(g)$, and if in the product $\alpha^{-1} f_{*}(s)$ some of the remnant of $f_{*}(s)$ cancels, then $g=s$. Similarly, if $\alpha$ is an initial segment of $f_{*}(g)$ and in the product $f_{*}(s) \alpha$ some of the remnant of $f_{*}(s)$ cancels, then $g^{-1}=s$.

The example below shows that Lemma 7.1 cannot, as it stands, be applied to iterates of standard representatives. Thus Wagner's proof breaks down in our setting.

ExAmple 7.2. For $X$ equal to the wedge of three circles, let $f$ be the standard representative for the homomorphism given by $f_{*}(a)=b c a, f_{*}(b)=$ $A B B$ and $f_{*}(c)=c$. The second iterate is given below, first in its unreduced 
form and then in its reduced form with the remnant for $f_{*}^{2}$ underlined:

$$
\begin{aligned}
& f_{*}^{2}(a)=A B B \cdot c \cdot b c a=A B B \underline{c b} c a, \\
& f_{*}^{2}(b)=A C B \cdot b b a \cdot b b a=A C \underline{b a} b b a, \\
& f_{*}^{2}(c)=c=\underline{c} .
\end{aligned}
$$

Let $y$ be the fixed point that corresponds to the $B$ in this unreduced version of $f_{*}^{2}(b)$. Then $y$ has address 41 and itinerary $(4,1,4)$. We have $W_{x}^{-1}=A C B$, which is of course an initial segment of the unreduced version of $f_{*}^{2}(b)$.

Our problem is that $W_{x}^{-1}$ is not an initial segment of the reduced version of $f_{*}^{2}(b)$ and that in the product $W_{x} f_{*}^{2}(A)$ we have remnant of $f_{*}^{2}(A)$ canceling, even though $b \neq A^{-1}$. Thus Lemma 7.1 does not hold for iterates of standard representatives. We need a lemma which will allow us to consider Wagner tails that occur as initial segments of unreduced image words. This phenomenon occurs only for iterates of our standard representative. This explains the significance of our earlier statement that the iterates of standard representatives are not the standard representatives of iterates.

7.1. The solution: using subremnant. We solve the problem by replacing remnant in Lemma 7.1 with a new concept, "subremnant." We state here our results and postpone both the definition of subremnant, which is technical, and a proof of Lemma 7.3 to Section 7.2. The proof of Theorem 3.3 is left to the reader because it is exactly the same as that of Theorem 2.12 if Lemma 7.1 is replaced by Lemma 7.3.

LEMMA 7.3. Let $f$ be a standard representative for a homomorphism $h: G \rightarrow G$ with remnant. Let $\alpha \in G$, and let $g, s \in \mathcal{G}^{ \pm}$.

If $\alpha$ is an initial segment of the unreduced version of $f_{*}^{n}(g)$, and if in the product $\alpha^{-1} f_{*}^{n}(s)$ some of the subremnant of $f_{*}^{n}(s)$ cancels, then $g=s$.

Similarly, if $\alpha$ is an initial segment of the unreduced version $f_{*}^{n}(g)$, and if in the product $f_{*}^{n}(s) \alpha$ some of the subremnant of $f_{*}^{n}(s)$ cancels, then $g^{-1}=s$.

7.2. Definition of subremnant, and proof of Lemma 7.3. Let $f$ be a standard representative with remnant. For each $s \in \mathcal{G}^{ \pm}$, we express $f_{*}(s)$ as the reduced product $\theta_{s} \varrho_{s} \tau_{s}$ such that if $s \in \mathcal{G}^{ \pm}$then $\varrho_{s}$ is the remnant of $f_{*}(s)$, and if $s^{-1} \in \mathcal{G}^{ \pm}$then $\varrho_{s}$ is the inverse of the remnant of $f_{*}\left(s^{-1}\right)$. We require that the product $\theta_{s} \varrho_{s} \tau_{s}$ be reduced except that $\theta_{s}$ and $\tau_{s}$ might be trivial. For any $s, t \in \mathcal{G}^{ \pm}$such that $s \neq t^{-1}$, in the product $f_{*}(s) f_{*}(t)=$ $\theta_{s} \varrho_{s} \tau_{s} \cdot \theta_{t} \varrho_{t} \tau_{t}$ the only place where cancellation can occur is at the dot. In particular neither $\varrho_{s}$ nor $\varrho_{t}$ have any cancellation. 
We define the subremnant for $f_{*}^{n}(s)$ (denoted $\hat{\varrho}_{n, s}$ ) recursively. Firstly, the subremnant of $f_{*}(s)\left(=f_{*}^{1}(s)\right)$ is just the usual remnant, so $\varrho_{1, s}=\varrho_{s} \neq 1$. Next we write $\varrho_{s}=r_{1} \cdots r_{y}$ as a reduced product of elements of $\mathcal{G}^{ \pm}$. We then define $\hat{\varrho}_{2, s}$ to be the unreduced version of $f_{*}\left(\varrho_{s}\right)$ with the first $\theta$ and the last $\tau$ removed. That is, $\varrho_{2, s}=\varrho_{r_{1}} \tau_{r_{1}} \theta_{r_{2}} \varrho_{r_{2}} \tau_{r_{2}} \cdots \theta_{r_{y}} \varrho_{r_{y}}$. Because $\varrho_{s} \neq 1$, each $\varrho_{r_{i}} \neq 1$. Thus whenever $f$ has remnant, $f^{2}$ has subremnant.

Next, we have $f_{*}^{2}(s)=f_{*}\left(\theta_{s} \varrho_{s} \tau_{s}\right)=f_{*}\left(\theta_{s}\right) \theta_{r_{1}} \hat{\varrho}_{2, s} \tau_{r_{y}} f_{*}\left(\tau_{s}\right)$. Define $\hat{\theta}_{2, s}$ to be $f_{*}\left(\theta_{s}\right) \theta_{r_{1}}$, and $\hat{\tau}_{2, s}$ to be $\tau_{r_{y}} f_{*}\left(\tau_{s}\right)$. In this way, $f_{*}^{2}(s)$ in its unreduced form is expressed as $f_{*}^{2}(s)=\hat{\theta}_{2, s} \hat{\varrho}_{2, s} \hat{\tau}_{2, s}$. And of course $\hat{\varrho}_{2, s} \neq 1$.

The inductive step is very similar to the second. More precisely, for $n>2$ define $\hat{\varrho}_{n, s}:=\hat{\varrho}_{n-1, r_{1}} \hat{\tau}_{n-1, r_{1}} \hat{\theta}_{n-1, r_{2}} \hat{\varrho}_{n-1, r_{2}} \hat{\tau}_{n-1, r_{2}} \cdots \hat{\theta}_{n-1, r_{y}} \hat{\varrho}_{n-1, r_{y}}$, the subword of the unreduced form of $f_{*}^{n}(s)$. Again, because each $\hat{\varrho}_{n-1, r_{i}} \neq 1$, we have $\hat{\varrho}_{n, s} \neq 1$. We have proven the following extension of Lemma 2.11:

LEMMA 7.4. Let $f$ be a standard representative of a homomorphism with remnant. Then any iterate of $f$ has subremnant, meaning that $\hat{\varrho}_{n, s} \neq 1$ for each $n \in \mathbb{N}$ and $s \in \mathcal{G}^{ \pm}$.

To make this a little clearer, we note that

$$
\begin{aligned}
f_{*}^{n}(s)= & f_{*}^{n-1}\left(\theta_{s} \varrho_{s} \tau_{s}\right)=f_{*}^{n-1}\left(\theta_{s}\right) f_{*}^{n-1}\left(r_{1} \cdots r_{y}\right) f_{*}^{n-1}\left(\tau_{s}\right) \\
= & f_{*}^{n-1}\left(\theta_{s}\right) \hat{\theta}_{n-1, r_{1}} \cdot \hat{\varrho}_{n-1, r_{1}} \hat{\tau}_{n-1, r_{1}} \hat{\theta}_{n-1, r_{2}} \hat{\varrho}_{n-1, r_{2}} \hat{\tau}_{n-1, r_{2}} \\
& \cdots \hat{\theta}_{n-1, r_{y}} \hat{\varrho}_{n-1, r_{y}} \cdot \hat{\tau}_{n-1, r_{y}} f_{*}^{n-1}\left(\tau_{s}\right) .
\end{aligned}
$$

Note that $\hat{\varrho}_{n, s}$ is the unreduced version of $f_{*}^{n-1}\left(\varrho_{s}\right)$, with $\hat{\theta}_{n-1, r_{1}}$ and $\hat{\tau}_{n-1, r_{y}}$ removed. As above, we define $\hat{\theta}_{n, s}$ to be $f_{*}^{n-1}\left(\theta_{s}\right) \hat{\theta}_{n-1, r_{1}}$, and define $\hat{\tau}_{n, s}$ to be $\hat{\tau}_{n-1, r_{y}} f_{*}^{n-1}\left(\tau_{s}\right)$. We have $f_{*}^{n}(s)=\hat{\theta}_{n, s} \hat{\varrho}_{n, s} \hat{\tau}_{n, s}$, and we define $\hat{\varrho}_{n, s}$ to be the subremnant of the unreduced form of $f_{*}^{n}(s)$.

Finally, we sketch the proof of Lemma 7.3. We begin by defining $p(n, s)$ and $q(n, s)$ : For any $s \in \mathcal{G}^{ \pm}$, suppose that $\varrho_{s}=r_{1} \cdots r_{k}$ is a reduced product of elements of $\mathcal{G}^{ \pm}$. Define $p(s)$ and $q(s)$ by $p(s):=r_{1}$ and $q(s):=r_{k}$. For any $k>1$, define $p(k, s)$ to be the first letter of the unreduced version of $\hat{\varrho}_{k, s}$. We will prove below that this is also the first letter of the reduced version of $\hat{\varrho}_{k, s}$. Similarly, for any $k>1$, define $q(k, s)$ to be the last letter of the unreduced version of $\hat{\varrho}_{k, s}$. The proof of the following is left to the reader:

FACTS.

1. $p(s)=p(1, s)$ by definition.

2. $p(p(s))=p(2, s)$ and in general, by induction, $p^{k}(s)=p(k, s)$.

3. $p(k, p(s))=p(k+1, s)$.

Similar facts hold for $q(k, s)$. 
Lemma 7.3 follows directly from the following technical lemma.

LEMma 7.5. Let $n$ be a fixed natural number and let $f$ be a standard representative of a homomorphism with remnant.

1. For any $s \in \mathcal{G}^{ \pm}$, when the unreduced version of $f_{*}^{n}(s)$ is reduced, the first and last letter of the subremnant of $f_{*}^{n}(s)$ do not cancel. That is, $p(n, s)$ and $q(n, s)$ do not cancel when the unreduced version of $f_{*}^{n}(s)$ is reduced.

2. Thus $p(n, s)$ is the first letter and $q(n, s)$ is the last letter of the reduced version of $\hat{\varrho}_{n, s}$.

3. In addition, for any reduced word $a_{1} \cdots a_{k}$ with each $a_{i} \in \mathcal{G}^{ \pm}$, when the product $f_{*}^{n}\left(a_{1} \cdots a_{k}\right)$ is reduced, for each $i$ the letters $p\left(n, a_{i}\right)$ and $q\left(n, a_{i}\right)$ do not cancel.

Proof. We prove the three statements together by induction.

For $n=1$, the subremnant is the remnant, and the unreduced version of $f_{*}(s)$ is the same string as the reduced version. Thus the first statement holds, by Wagner's work. By the definition of remnant, the second and third statements hold as well.

Let $f_{*}(s)=\theta_{s} \varrho_{s} \tau_{s}=s_{1} \cdots s_{k}$ be reduced, with each $s_{i}$ in $\mathcal{G}^{ \pm}$. Then there exist $l$ and $m$ such that $s_{l}=p(s)$ and $s_{m}=q(s)$. Suppose that all three statements hold for $n$. Consider $f_{*}^{n+1}(s)=f_{*}^{n}\left(s_{1} \cdots s_{k}\right)$. For each $i$, by the third statement, $p\left(n, s_{i}\right)$ and $q\left(n, s_{i}\right)$ do not cancel when $f_{*}^{n}\left(s_{1} \cdots s_{k}\right)$ is reduced. We have $p\left(n, s_{l}\right)=p(n, p(s))=p(n+1, s)$, and we have proven that $p(n+1, s)$ does not cancel when $f_{*}^{n+1}(s)$ is reduced. The same proof works for $q(n+1, s)$. Thus the first two statements hold for $n+1$.

Next consider an arbitrary reduced product $a_{1} \cdots a_{k}$ with each $a_{i} \in \mathcal{G}^{ \pm}$. Then $f_{*}^{n+1}\left(a_{1} \cdots a_{k}\right)=f_{*}^{n}\left(f_{*}\left(a_{1} \cdots a_{k}\right)\right)$. For each $i$ the letters $p\left(a_{i}\right)$ and $q\left(a_{i}\right)$ do not cancel when $f_{*}\left(a_{1} \cdots a_{k}\right)$ is reduced. Let the reduced form of $f_{*}\left(a_{1} \cdots a_{k}\right)$ be $b_{1} \cdots b_{t}$ with $b_{j} \in \mathcal{G}^{ \pm}$. Each of the $p\left(n, a_{i}\right)$ and $q\left(n, a_{i}\right)$ occur in this product. We have $f_{*}^{n+1}\left(a_{1} \cdots a_{k}\right)=f_{*}^{n}\left(b_{1} \cdots b_{t}\right)$. By induction, for each $j$ we know that $p\left(n, b_{j}\right)$ and $q\left(n, b_{j}\right)$ do not cancel when $f_{*}^{n}\left(b_{1} \cdots b_{t}\right)$ is reduced. But for each $i$ we have, for some $j, p\left(n, b_{j}\right)=p\left(n, p\left(a_{i}\right)\right)=$ $p\left(n+1, a_{i}\right)$. The same is true for the $q$ 's. Thus when $f_{*}^{n+1}\left(a_{1} \cdots a_{k}\right)$ is reduced, we know that $p\left(n+1, a_{i}\right)$ and $q\left(n+1, a_{i}\right)$ do not cancel for all $i$.

\section{References}

[1] M. Bestvina and M. Handel, Train-tracks for surface homeomorphisms, Topology 34 (1995), 109-140. 
[2] W. Bosma, J. J. Cannon and G. Mathews, Programming with algebraic structures: Design of the Magma language, in: M. Giesbrecht (ed.), Proc. 1994 Int. Symposium on Symbolic and Algebraic Computation (Oxford, 1994), Assoc. Comput. Mach., 1994, 52-57.

[3] R. F. Brown, The Lefschetz Fixed Point Theorem, Scott, Foresman and Co., 1971.

[4] E. Fadell and S. Husseini, The Nielsen number on surfaces, in: Contemp. Math. 21, Amer. Math. Soc., 1983, 59-98.

[5] E. Hart, Algebraic techniques for calculating the Nielsen number on hyperbolic surfaces, in: Handbook of Topological Fixed Point Theory, Kluwer, 2005, 463-487.

[6] - Reidemeister conjugacy for finitely generated free fundamental groups, Fund. Math. 199 (2008), 93-118.

[7] E. Hart, P. R. Heath and E. Keppelmann, Algorithms for Nielsen type periodic numbers of maps with remnant on surfaces with boundary and on bouquets of circles $I I$, in preparation.

[8] E. Hart and E. Keppelmann, Explorations in Nielsen periodic point theory for the double torus, Topology Appl. 95 (1999), 1-30.

[9] —, - Nielsen periodic point theory for periodic maps on orientable surfaces, ibid. 153 (2006), 1399-1420.

[10] P. Heath and E. Keppelmann, Fibre techniques in Nielsen periodic point theory on nil and solvmanifolds. I, ibid. 76 (1997), 217-247.

[11] P. Heath, R. Piccinini and C. Y. You, Nielsen-type numbers for periodic points. I, in: Topological Fixed Point Theory and Applications (Tianjin, 1988), Lecture Notes in Math. 1411, Springer, Berlin, 1989, 88-106.

[12] P. Heath and C. Y. You, Nielsen-type numbers for periodic points. II, Topology Appl. 43 (1992), 219-236.

[13] B. J. Jiang, Lectures on Nielsen Fixed Point Theory, Contemp. Math. 14, Amer. Math Soc., Providence, RI, 1983.

[14] M. Kelly, Minimizing the number of fixed points for selfmaps of surfaces with boundary, Pacific J. Math. 126 (1987), 81-123.

[15] —, Computing Nielsen numbers of surface homeomorphisms, Topology 35 (1996) $13-25$.

[16] —, Nielsen fixed point theory on surfaces, in: Handbook of Topological Fixed Point Theory, Kluwer, 2005, 647-658.

[17] S. W. Kim, Computation of Nielsen numbers for maps of compact surfaces with boundary, J. Pure Appl. Algebra 208 (2007), 467-479.

[18] —, Nielsen numbers of maps of polyhedra with fundamental group free on two generators, in preparation.

[19] J. Llibre and A. Nunes, Minimum number of fixed points for maps of the figure eight space, in: Discrete Dynamical Systems, Int. J. Bifur. Chaos Appl. Sci. Engrg. 9 (1999), 1795-1802.

[20] C. McCord, Computing Nielsen Numbers, in: Nielsen Theory and Dynamical Systems, Contemp. Math. 152, Amer. Math. Soc., 1993, 249-267.

[21] J. Wagner, An algorithm for calculating the Nielsen number on surfaces with boundary, Trans. Amer. Math. Soc. 351 (1999), 41-62.

[22] —, Classes of Wecken maps of surfaces with boundary, Topology Appl. 76 (1997), $27-46$. 
[23] P. Yi, An algorithm for computing the Nielsen number of maps on the pants surface, Ph.D. thesis, UCLA, 2003.

Department of Mathematics

Department of Mathematics

Colgate University

13 Oak Drive

Hamilton, NY 13346-1398, U.S.A.

E-mail: ehart@colgate.edu

Department of Mathematics and Statistics MS084

University of Nevada

Reno, NV 89557, U.S.A.

E-mail: keppelma@unr.edu

Received 12 June 2006;

in revised form 10 August 2007 and 20 March 2008 